\title{
Decay estimates for Schrödinger heat semigroup with inverse square potential in Lorentz spaces
}

\author{
Kazuhiro Ishige and Yujiro Tateishi \\ Graduate School of Mathematical Sciences, The University of Tokyo \\ 3-8-1 Komaba, Meguro-ku, Tokyo 153-8914, Japan \\ E-mail addresses: \\ ishige@ms.u-tokyo.ac.jp (K. Ishige), tateishi@ms.u-tokyo.ac.jp (Y. Tateishi)
}

\begin{abstract}
Let $H:=-\Delta+V$ be a nonnegative Schrödinger operator on $L^{2}\left(\mathbf{R}^{N}\right)$, where $N \geq 2$ and $V$ is an inverse square potential. In this paper we obtain sharp decay estimates of the operator norms of $e^{-t H}$ and $\nabla e^{-t H}$ in Lorentz spaces.
\end{abstract}




\section{Introduction}

Nonnegative Schrödinger operators $H:=-\Delta+V$ on $L^{2}\left(\mathbf{R}^{N}\right)$ and their heat semigroups $e^{-t H}$ have been studied by many mathematicians since the pioneering work due to Simon [36]. See e.g. [1], [3], 4], 6], [11, [12], [14]-[17], 23]- 29], 31]-38] and references therein. (See also the monographs of Davies [5], Grigor'yan [8] and Ouhabaz [30.) Decay estimates of operator norms of $\nabla^{\ell} e^{-t H}$ are fundamental to the study of nonnegative Schrödinger operators and their related fields. Here $\nabla:=\left(\partial / \partial x_{1}, \ldots, \partial / \partial x_{N}\right)$ and $\ell \in\{0,1, \ldots\}$. However, the large time decay estimates of operator norms of $\nabla^{\ell} e^{-t H}$ are delicate and they are widely open even in Lebesgue spaces (see e.g. [6], [13]-[15], 24], [36] and references therein).

In this paper we focus on a nonnegative Schrödinger operator $H:=-\Delta+V$, where $V$ is an inverse square potential, more precisely, $V$ satisfies the following condition $(\mathrm{V})$ :

$$
\begin{cases}\text { (i) } \quad & V=V(|x|) \text { in } \mathbf{R}^{N} \backslash\{0\} \text { and } V \in C^{1}((0, \infty)) ; \\ \text { (ii) } \quad & V(r)=\lambda_{1} r^{-2}+O\left(r^{-2+\rho_{1}}\right) \text { as } r \rightarrow+0, \\ & V(r)=\lambda_{2} r^{-2}+O\left(r^{-2-\rho_{2}}\right) \text { as } r \rightarrow \infty, \\ & \text { for some } \lambda_{1}, \lambda_{2} \in\left[\lambda_{*}, \infty\right) \text { with } \lambda_{*}:=-(N-2)^{2} / 4 \text { and } \rho_{1}, \rho_{2}>0 ; \\ \text { (iii) } & \sup _{r>0}\left|r^{3} \frac{d}{d r} V(r)\right|<\infty .\end{cases}
$$

The purpose of this paper is to obtain the decay estimates of

$$
\left\|\nabla^{\ell} e^{-t H}\right\|_{\left(L^{p, \sigma} \rightarrow L^{q, \theta}\right)}:=\sup \left\{\left\|\nabla^{\ell} e^{-t H} \phi\right\|_{L^{q, \theta}\left(\mathbf{R}^{N}\right)}: \phi \in C_{\mathrm{c}}\left(\mathbf{R}^{N}\right) \text { with }\|\phi\|_{L^{p, \sigma}\left(\mathbf{R}^{N}\right)}=1\right\},
$$

where $\ell \in\{0,1\}$ and

$$
(p, q, \sigma, \theta) \in \Lambda:=\left\{\begin{array}{l}
\sigma=1 \text { if } p=1, \quad \sigma=\infty \text { if } p=\infty \\
1 \leq p \leq q \leq \infty, \sigma, \theta \in[1, \infty]: \quad \theta=1 \text { if } q=1, \quad \theta=\infty \text { if } q=\infty \\
\sigma \leq \theta \text { if } p=q
\end{array}\right\} .
$$

Here $e^{-t H} \phi$ is a solution to the Cauchy problem

$$
\begin{cases}\partial_{t} u=\Delta u-V(|x|) u & \text { in } \quad \mathbf{R}^{N} \times(0, \infty), \\ u(\cdot, 0)=\phi & \text { in } \quad \mathbf{R}^{N}\end{cases}
$$

and $\left\|\nabla^{\ell} e^{-t H}\right\|_{\left(L^{p, \sigma} \rightarrow L^{q, \theta}\right)}$ is the operator norm of $\nabla^{\ell} e^{-t H}$ from the Lorentz space $L^{p, \sigma}\left(\mathbf{R}^{N}\right)$ to $L^{q, \theta}\left(\mathbf{R}^{N}\right)$. This paper can be regarded as a continuation of [12], where the large time sharp decay estimates of $\left\|e^{-t H}\right\|_{\left(L^{p, \sigma} \rightarrow L^{q, \theta}\right)}$ were obtained when $V \in C^{1}([0, \infty))$ and $\lambda_{2}>\lambda_{*}$.

We introduce some notations. For $x \in \mathbf{R}^{N}$ and $R>0$, let $B(x, R):=\left\{y \in \mathbf{R}^{N}:|y-x|<R\right\}$ and $B(x, R)^{c}:=\mathbf{R}^{N} \backslash B(x, R)$. For any $r \in[1, \infty]$, let $r^{\prime}$ be the Hölder conjugate number of $r$, that is,

$$
r^{\prime}=\frac{r}{r-1} \quad \text { if } \quad 1<r<\infty, \quad r^{\prime}=1 \quad \text { if } \quad r=\infty, \quad r^{\prime}=\infty \quad \text { if } \quad r=1 .
$$


Let $\Delta_{\mathbf{S}^{N-1}}$ be the Laplace-Beltrami operator on $\mathbf{S}^{N-1}$. Let $\left\{\omega_{k}\right\}_{k=0}^{\infty}$ be the eigenvalues of

$$
-\Delta_{\mathbf{S}^{N-1}} Q=\omega Q \quad \text { on } \quad \mathbf{S}^{N-1}, \quad Q \in L^{2}\left(\mathbf{S}^{N-1}\right) .
$$

Then $\omega_{k}=k(N+k-2)$ for $k \in\{0,1,2, \ldots\}$. Let $\left\{Q_{k, i}\right\}_{i=1}^{d_{k}}$ and $d_{k}$ be the orthonormal system and the dimension of the eigenspace corresponding to $\omega_{k}$, respectively. Here

$$
d_{k}=\frac{(N+2 k-2)(N+k-3) !}{(N-2) ! k !}=O\left(k^{N-2}\right) \quad \text { as } \quad k \rightarrow \infty .
$$

Assume condition (V) and let $H:=-\Delta+V$ be nonnegative, that is,

$$
\int_{\mathbf{R}^{N}}\left[|\nabla \phi|^{2}+V(|x|) \phi^{2}\right] d x \geq 0, \quad \phi \in C_{c}^{\infty}\left(\mathbf{R}^{N} \backslash\{0\}\right) .
$$

The operator $H$ is said subcritical if, for any $W \in C_{c}\left(\mathbf{R}^{N}\right), H-\epsilon W$ is nonnegative for small enough $\epsilon>0$. If not, the operator $H$ is said critical.

For any $k \in\{0,1,2, \ldots\}$, set $A_{1, k}:=A_{\lambda_{1}+\omega_{k}}^{+}$and

$$
\begin{aligned}
A_{2, k} & := \begin{cases}A_{\lambda_{2}}^{-} & \text {if } k=0 \text { and } H \text { is critical, } \\
A_{\lambda_{2}+\omega_{k}}^{+} & \text {otherwise },\end{cases} \\
B_{k} & :=\left\{\begin{array}{ll}
1 & \text { if } k=0, \lambda_{2}=\lambda_{*} \\
0 & \text { otherwise }
\end{array}\right. \text { and is subcritical, }
\end{aligned}
$$

Here

$$
A_{\lambda}^{ \pm}:=\frac{-(N-2) \pm \sqrt{D_{\lambda}}}{2} \text { for } \lambda \geq \lambda_{*}, \text { where } D_{\lambda}:=(N-2)^{2}+4 \lambda .
$$

By the standard theory for ordinary differential equations we see that, for any $k \in\{0,1,2, \ldots\}$, there exists a unique solution $h_{k}=h_{k}(r)$ to the problem

$$
\begin{aligned}
& \frac{d^{2}}{d r^{2}} h_{k}+\frac{N-1}{r} \frac{d}{d r} h_{k}-V_{k}(r) h_{k}=0 \quad \text { in }(0, \infty), \\
& h_{k}(r)=r^{A_{1, k}}(1+o(1)) \quad \text { as } \quad r \rightarrow+0
\end{aligned}
$$

where $V_{k}(r):=V(r)+\omega_{k} r^{-2}$. (See also Section 2.1.) Notice that $h_{k} \in L^{2}(B(0,1))$. Furthermore, it follows from [17, Theorem 1.1] that

$$
h_{k}(r)=c_{k} v_{k}(r)(1+o(1)) \quad \text { as } \quad r \rightarrow \infty, \quad \text { where } \quad v_{k}(r):=r^{A_{2, k}}(\log r)^{B_{k}},
$$

for some $c_{k}>0$. The function $h_{0}$ is said a positive harmonic function for the operator $H$ and it plays an important role in our analysis. When $H$ is critical, if $h_{0} \notin L^{2}\left(\mathbf{R}^{N}\right)$, then $H$ is said null-critical: if not, $H$ is said positive-critical. The decay of the fundamental solution $p=p(x, y, t)$ corresponding to $e^{-t H}$ depends on whether $H$ is either subcritical, null-critical or positive critical. See [31]. (See also [17].) In this paper we assume either

(i) $H$ is subcritical or (ii) $H$ is critical and $A_{2,0}>-N / 2$, 
and obtain decay estimates of $\left\|\nabla^{\ell} e^{-t H}\right\|_{\left(L^{p, \sigma} \rightarrow L^{q, \theta}\right)}$ as $t \rightarrow+0$ and as $t \rightarrow \infty$, where $\ell \in\{0,1\}$ and $(p, q, \sigma, \theta) \in \Lambda$. Case (ii) is in the null-critical one. We remark that $e^{-t H} \phi$ does not necessarily decay as $t \rightarrow \infty$ if $H$ is positive-critical. (See also Remark 1.1 (iii).)

Now we are ready to state the main results of this paper.

Theorem 1.1 Assume conditions (V) and (N'). Let $(p, q, \sigma, \theta) \in \Lambda, j \in\{0,1,2, \ldots\}$ and $\ell \in$ $\{0,1\}$. Then there exists $C>0$ such that

$$
\left\|\partial_{t}^{j} \nabla^{\ell} e^{-t H}\right\|_{\left(L^{p, \sigma} \rightarrow L^{q, \theta}\right)} \leq C t^{-\frac{N}{2}-j} \frac{\left\|h_{0}\right\|_{L^{p^{\prime}, \sigma^{\prime}}(B(0, \sqrt{t}))}}{h_{0}(\sqrt{t})}\left(\frac{\left\|\nabla^{\ell} h_{0}\right\|_{L^{q, \theta}(B(0, \sqrt{t}))}}{h_{0}(\sqrt{t})}+t^{\frac{N}{2 q}-\frac{\ell}{2}}\right)
$$

for $t>0$.

We remark that the right-hand side of inequality (1.6) possibly diverges for some $(p, q, \sigma, \theta) \in \Lambda$. As a corollary of Theorem 1.1, we have:

Corollary 1.1 Assume conditions (V) and (N'). Let $(p, q, \sigma, \theta) \in \Lambda$. Then there exists $C>0$ such that

$$
\left\|e^{-t H}\right\|_{\left(L^{p, \sigma} \rightarrow L^{q, \theta}\right)} \leq C t^{-\frac{N}{2}} \frac{\left\|h_{0}\right\|_{L^{p^{\prime}, \sigma^{\prime}}(B(0, \sqrt{t}))}\left\|h_{0}\right\|_{L^{q, \theta}(B(0, \sqrt{t}))}}{h_{0}(\sqrt{t})^{2}} \text { for } \quad t>0 .
$$

Remark 1.1 (i) Assume conditions (V) and (N'). Furthermore, assume that $V \in C^{1}([0, \infty))$ and $\lambda_{2}>\lambda_{*}$. Then the large time sharp decay estimates of $\left\|e^{-t H}\right\|_{\left(L^{p, \sigma} \rightarrow L^{q, \theta}\right)}$ have been already obtained in [12, Theorem 1.1]. Our decay estimate (1.7) gives the same decay estimate as in [12, Theorem 1.1] and it has a simpler expression.

(ii) In a forthcoming paper [19], under conditions (V) and (N'), we obtain decay estimates of $\left\|\nabla^{\ell} e^{-t H}\right\|_{\left(L^{p, \sigma} \rightarrow L^{q, \theta}\right)}$ for $\ell \in\{0,1,2, \ldots\}$. Furthermore, we find $C>0$ such that

$$
\left\|\nabla^{\ell} e^{-t H}\right\|_{\left(L^{\left.p, \sigma \rightarrow L^{q, \theta}\right)}\right.} \geq C^{-1} t^{-\frac{N}{2}} \frac{\left\|h_{0}\right\|_{L^{p^{\prime}, \sigma^{\prime}}(B(0, \sqrt{t}))}}{h_{0}(\sqrt{t})}\left(\frac{\left\|\nabla^{\ell} h_{0}\right\|_{L^{q, \theta}(B(0, \sqrt{t}))}}{h_{0}(\sqrt{t})}+t^{\frac{N}{2 q}-\frac{\ell}{2}}\right)
$$

for $t>0$, where $\ell \in\{0,1\}$, and show the sharpness of decay estimate (1.6).

(iii) Under condition (V), case (ii) covers all of the null-critical cases except for the case when $A_{2,0}=-N / 2$. On the other hand, in this paper, we don't treat the case when $A_{2,0}=-N / 2$ since it is on the borderline between null-criticality and positive-criticality and it is too delicate.

We prove Theorem 1.1 by developing the arguments in [14] and combining the results of [17] with parabolic regularity theorems. For any $\phi \in C_{c}\left(\mathbf{R}^{N}\right)$, we find radially symmetric functions $\left\{\phi_{k, i}\right\}_{k=0,1, \ldots, i=1, \ldots, d_{k}} \subset L^{2}\left(\mathbf{R}^{N}\right)$ such that

$$
\phi(x)=\sum_{k=0}^{\infty} \sum_{i=1}^{d_{k}} \phi_{k, i}(|x|) Q_{k, i}\left(\frac{x}{|x|}\right) \quad \text { in } \quad L^{2}\left(\mathbf{R}^{N}\right) .
$$


Let $H_{k}:=-\Delta+V_{k}(|x|)$ and set

$$
v_{k, i}(|x|, t):=\left[e^{-t H_{k}} \phi_{k, i}\right](|x|), \quad u_{k, i}(x, t)=v_{k, i}(|x|, t) Q_{k, i}\left(\frac{x}{|x|}\right) .
$$

Then

$$
\left[e^{-t H} \phi\right](x)=\sum_{k=0}^{\infty} \sum_{i=1}^{d_{k}} u_{k, i}(x, t)=\sum_{k=0}^{\infty} \sum_{i=1}^{d_{k}} v_{k, i}(|x|, t) Q_{k, i}\left(\frac{x}{|x|}\right) \quad \text { in } \quad C^{2}(K)
$$

for compact sets $K \subset \mathbf{R}^{N} \backslash\{0\}$ and $t>0$ (see [18] and [16]). For the proof of Theorem [1.1, we obtain uniform estimates of $\left\{v_{k, i}\right\}$ inside parabolic cones by constructing supersolutions. This is the main difficulty for the proof of Theorem 1.1 and requires delicate analysis. Then, due to the radial symmetry of $v_{k, i}$ and parabolic regularity theorems, we obtain decay estimates of $\nabla^{\ell} e^{-t H} \phi$ inside parabolic cones. On the other hand, we obtain estimates of $\nabla^{\ell} e^{-t H} \phi$ outside parabolic cones by the Gaussian estimate of the fundamental solution $p=p(x, y, t)$ and parabolic regularity theorems. Combining the estimates of $\nabla^{\ell} e^{-t H} \phi$ inside and outside parabolic cones, we obtain Theorem 1.1 .

The rest of this paper is organized as follows. In Section 2 we formulate a definition of the solution to problem (P) and Lorentz spaces. Furthermore, we study the asymptotic behavior of $h_{k}$ as $r \rightarrow+0$ and as $r \rightarrow \infty$. In Section 3 we obtain upper estimates of $e^{-t H} \phi$ by using the Gaussian estimate of $p=p(x, y, t)$. In Section 4, combining the estimates in Sections 2 and 3, we obtain uniform estimates of $\left\{v_{k, i}\right\}$ inside parabolic cones with respect to $k$ and $i$. In Section 5 we complete the proof of Theorem 1.1. Furthermore, we prove Corollary 1.1 .

\section{Preliminaries}

In this section we formulate a definition of the solution to problem $(\mathrm{P})$ and introduce Lorentz spaces. We also obtain uniform estimates of the solution $h_{k}$ to problem (1.4) with respect to $k$.

Throughout this paper, for any positive functions $f$ and $g$ on a set $E$, we write $f \asymp g$ for $x \in E$ if there exists $c>0$ such that $c^{-1} \leq f(x) / g(x) \leq c$ for $x \in E$. By the letter $C$ we denote generic positive constants and they may have different values also within the same line.

\subsection{Definition of solutions to problem $(\mathrm{P})$ and Lorentz spaces}

Before considering problem $(\mathrm{P})$, we formulate a definition of the solution to the problem

$$
\begin{cases}\partial_{t} w=\frac{1}{\nu} \operatorname{div}(\nu \nabla w) & \text { in } \quad \mathbf{R}^{N} \times(0, \infty), \\ w(\cdot, 0)=\phi_{*} & \text { in } \quad \mathbf{R}^{N}\end{cases}
$$

where $\nu:=h_{0}^{2}$ and $\phi_{*} \in L^{2}\left(\mathbf{R}^{N}, \nu d x\right)$.

Definition 2.1 Let $\phi_{*} \in L^{2}\left(\mathbf{R}^{N}, \nu d x\right)$. A measurable function $w$ in $\mathbf{R}^{N} \times(0, \infty)$ is said a solution to problem $(\mathrm{W})$ if

$$
w \in L^{\infty}\left(0, \infty: L^{2}\left(\mathbf{R}^{N}, \nu d x\right)\right) \cap L^{2}\left(0, \infty: H^{1}\left(\mathbf{R}^{N}, \nu d x\right)\right)
$$


and $w$ satisfies

$$
\int_{\mathbf{R}^{N}} \psi(x, 0) \phi_{*}(x) \nu(x) d x+\int_{0}^{\infty} \int_{\mathbf{R}^{N}}\left\{-w \partial_{t} \psi+\nabla w \nabla \psi\right\} \nu(x) d x d t=0
$$

for $\psi \in C_{\mathrm{c}}^{\infty}\left(\mathbf{R}^{N} \times[0, \infty)\right)$.

Problem (W) possesses a unique solution $w$ such that

$$
\|w(t)\|_{L^{2}\left(\mathbf{R}^{N}, \nu d x\right)} \leq\left\|\phi_{*}\right\|_{L^{2}\left(\mathbf{R}^{N}, \nu d x\right)} \quad \text { for } \quad t>0 .
$$

See [18, Section 2.1]. We denote by $e^{-t H_{*}} \phi_{*}$ the unique solution to problem (W).

Definition 2.2 Let $\phi \in L^{2}\left(\mathbf{R}^{N}\right)$. Set

$$
\left[e^{-t H} \phi\right](x):=h_{0}(|x|)\left[e^{-t H_{*}} \phi_{*}\right](x) \quad \text { with } \quad \phi_{*}(x):=\frac{\phi(x)}{h_{0}(|x|)}
$$

for $x \in \mathbf{R}^{N}$ and $t>0$. Then the function $e^{-t H} \phi$ is said a solution to problem $(\mathrm{P})$.

Notice that $\phi \in L^{2}\left(\mathbf{R}^{N}\right)$ if and only if $\phi_{*} \in L^{2}\left(\mathbf{R}^{N}, \nu d x\right)$.

We define the Lorentz spaces. For any measurable function $\phi$ in $\mathbf{R}^{N}$, we denote by $\mu=\mu(\lambda)$ the distribution function of $\phi$, that is,

$$
\mu(\lambda):=\left|\left\{x \in \mathbf{R}^{N}:|\phi(x)|>\lambda\right\}\right| \text { for } \quad \lambda>0 .
$$

Here $|E|$ is the $N$-dimensional Lebesgue measure of $E$ for measurable sets $E$ in $\mathbf{R}^{N}$. We define the non-increasing rearrangement $\phi^{*}$ of $\phi$ and the spherical rearrangement $\phi^{\sharp}$ of $\phi$ by

$$
\phi^{*}(s):=\inf \{\lambda>0: \mu(\lambda) \leq s\}, \quad \phi^{\sharp}(x):=\phi^{*}\left(\alpha_{N}|x|^{N}\right),
$$

for $s>0$ and $x \in \mathbf{R}^{N}$, respectively, where $\alpha_{N}$ is the volume of the unit ball in $\mathbf{R}^{N}$. For any $(p, p, \sigma, \sigma) \in \Lambda$, we define the Lorentz space $L^{p, \sigma}\left(\mathbf{R}^{N}\right)$ by

$$
L^{p, \sigma}\left(\mathbf{R}^{N}\right):=\left\{\phi: \phi \text { is measurable in } \mathbf{R}^{N},\|\phi\|_{L^{p, \sigma}}<\infty\right\},
$$

where

$$
\|\phi\|_{L^{p, \sigma}}:= \begin{cases}\left(\int_{\mathbf{R}^{N}}\left(|x|^{\frac{N}{p}} \phi^{\sharp}(x)\right)^{\sigma} \frac{d x}{|x|^{N}}\right)^{\frac{1}{\sigma}} & \text { if } \quad 1 \leq \sigma<\infty, \\ \sup _{x \in \mathbf{R}^{N}}|x|^{\frac{N}{p}} \phi^{\sharp}(x) & \text { if } \quad \sigma=\infty .\end{cases}
$$

Here $N / p=0$ if $p=\infty$. The Lorentz spaces have the following properties:

$$
\begin{array}{ll}
L^{p, p}\left(\mathbf{R}^{N}\right)=L^{p}\left(\mathbf{R}^{N}\right) & \text { if } 1 \leq p \leq \infty ; \\
L^{p, \sigma_{1}}\left(\mathbf{R}^{N}\right) \subset L^{p, \sigma_{2}}\left(\mathbf{R}^{N}\right) & \text { if } 1 \leq p<\infty \text { and } 1 \leq \sigma_{1} \leq \sigma_{2} \leq \infty .
\end{array}
$$


Furthermore, there exists $C>0$ depending only on $N$ such that

$$
\begin{array}{ll}
\|f+g\|_{L^{p, \sigma}} \leq C\left(\|f\|_{L^{p . \sigma}}+\|g\|_{L^{p, \sigma}}\right) & \text { if } f, g \in L^{p, \sigma}\left(\mathbf{R}^{N}\right), \\
\|f g\|_{L^{1}} \leq C\|f\|_{L^{p, \sigma}}\|g\|_{L^{p^{\prime}, \sigma^{\prime}}} & \text { if } f \in L^{p, \sigma}\left(\mathbf{R}^{N}\right), g \in L^{p^{\prime}, \sigma^{\prime}}\left(\mathbf{R}^{N}\right), \\
\|f * g\|_{L^{q, \theta}} \leq C\|f\|_{L^{p, \sigma}}\|g\|_{L^{r, s}} & \text { if } f \in L^{p, \sigma}\left(\mathbf{R}^{N}\right), g \in L^{r, s}\left(\mathbf{R}^{N}\right) .
\end{array}
$$

Here $(q, q, \theta, \theta),(r, r, s, s) \in \Lambda$ and

$$
\frac{1}{r}+\frac{1}{p}=\frac{1}{q}+1, \quad \frac{1}{\theta}=\frac{1}{s}+\frac{1}{\sigma}
$$

(See e.g. [2] and [7].) For any measurable function $f$ in a domain $\Omega$, we say that $f \in L^{p, \sigma}(\Omega)$ if and only if $\tilde{f} \in L^{p, \sigma}\left(\mathbf{R}^{N}\right)$, where $\tilde{f}$ is the zero extension of $f$ to $\mathbf{R}^{N}$. Furthermore, we write $\|f\|_{L^{p, \sigma}(\Omega)}=\|\tilde{f}\|_{L^{p, \sigma}}$. Then, for any $R>0$, the function $f_{A}$ defined by $f_{A}(x):=|x|^{A}$, where $A \in \mathbf{R}$, satisfies $f_{A} \in L^{p, \sigma}(B(0, R))$ if and only if

$$
p A+N>0 \text { for } 1 \leq \sigma<\infty, \quad p A+N \geq 0 \text { for } \sigma=\infty .
$$

Furthermore, under condition (2.3), we have

$$
\left\|f_{A}\right\|_{L^{p, \sigma}(B(0, \sqrt{t}))} \asymp t^{\frac{A}{2}+\frac{N}{2 p}} \quad \text { for } \quad t \in\left(0, R^{2}\right]
$$

In particular, for any $k \in\{0,1,2, \ldots\}$, by (1.4) we see that

$$
\frac{\left\|h_{k}\right\|_{L^{p, \sigma}(B(0, \sqrt{t}))}}{h_{k}(\sqrt{t})} \asymp t^{\frac{N}{2 p}} \quad \text { for } \quad 0<t \leq R^{2} \quad \text { if } \quad h_{k} \in L^{p . \sigma}(B(0,1)) .
$$

\subsection{Estimates of $h_{k}$}

Let $k \in\{0,1,2, \ldots\}$. Consider the ordinary differential equation

$$
\frac{d^{2}}{d r^{2}} h+\frac{N-1}{r} \frac{d}{d r} h-V_{k}(r) h=0 \quad \text { in } \quad(0, \infty)
$$

Then ODE (2.5) has two linearly independent solutions $h_{k}^{+}$and $h_{k}^{-}$such that

$$
h_{k}^{+}(r)=v_{k, \lambda_{1}}^{+}(r)(1+o(1)), \quad h_{k}^{-}(r)=v_{k, \lambda_{1}}^{-}(r)(1+o(1)),
$$

as $r \rightarrow+0$ and $h_{k}^{-}(1)=1$. (See e.g. [17].) Here

$$
v_{k, \lambda}^{+}(r):=r^{A_{\lambda+\omega_{k}}^{+},} v_{k, \lambda}^{-}(r):= \begin{cases}r^{-\frac{N-2}{2}}\left|\log \frac{r}{2}\right| & \text { if } \lambda=\lambda_{*} \text { and } k=0, \\ r^{A_{\lambda+\omega_{k}}^{-}} & \text {otherwise, }\end{cases}
$$

for $\lambda \geq \lambda_{*}$, where $A_{\lambda}^{ \pm}$is as in (1.3). The solution $h_{k}^{+}$coincides with $h_{k}$ (see (1.4)). We first prove the following proposition on the behavior of $h_{k}$ as $r \rightarrow+0$.

Proposition 2.1 Assume conditions (V) and (N'). Let $R \geq 1$. 
(a) For any $k \in\{0,1,2, \ldots\}$ and $\ell \in\{0,1\}$, there exists $C_{1}>0$ such that

$$
\left|\frac{d^{\ell}}{d r^{\ell}} h_{k}(r)-\frac{d^{\ell}}{d r^{\ell}} v_{k, \lambda_{1}}^{+}(r)\right| \leq C_{1} r^{-\ell+\rho_{1}} v_{k, \lambda_{1}}^{+}(r) \quad \text { for } \quad r \in(0, R] .
$$

(b) There exists $C_{2}>0$ and $k_{*} \in\{0,1,2, \ldots\}$ such that

$$
\left|h_{k}(r)-v_{k, \lambda_{1}}^{+}(r)\right| \leq C_{2} k^{-1} r^{\rho_{1}} v_{k, \lambda_{1}}^{+}(r), \quad C_{2}^{-1} k r^{-1} h_{k}(r) \leq \frac{d}{d r} h_{k}(r) \leq C_{2} k r^{-1} h_{k}(r),
$$

for $0<r \leq R$ and $k \in\left\{k_{*}, k_{*}+1, \ldots\right\}$.

For the proof, we prepare the following lemma.

Lemma 2.1 Let $k \in\{0,1,2, \ldots\}, \lambda \geq \lambda_{*}$ and $R>0$. Let $f$ be a continuous function in $(0, R]$ such that

$$
|f(r)| \leq r^{-2+\epsilon} v_{k, \lambda}^{+}(r), \quad r \in(0, R],
$$

for some $\epsilon>0$. Set

$$
F_{k, \lambda}^{+}[f](r):=v_{k, \lambda}^{+}(r) \int_{0}^{r} s^{1-N}\left[v_{k, \lambda}^{+}(s)\right]^{-2}\left(\int_{0}^{s} \tau^{N-1} v_{k, \lambda}^{+}(\tau) f(\tau) d \tau\right) d s
$$

for $r \in(0, R]$. Then there exists $C>0$, independent of $k$, such that

$$
\left|\frac{d^{\ell}}{d r^{\ell}} F_{k, \lambda}^{+}[f](r)\right| \leq C(k+1)^{\ell-1} r^{-\ell+\epsilon} v_{k, \lambda}^{+}(r) \quad \text { for } \quad r \in(0, R]
$$

where $\ell \in\{0,1\}$. Furthermore,

$$
\frac{d^{2}}{d r^{2}} F_{k, \lambda}^{+}[f](r)+\frac{N-1}{r} \frac{d}{d r} F_{k, \lambda}^{+}[f](r)-\frac{\lambda+\omega_{k}}{r^{2}} F_{k, \lambda}^{+}[f](r)=f(r) \quad \text { in } \quad(0, R] .
$$

Proof. Assume (2.6) for some $\epsilon>0$. Since $\lambda \geq \lambda_{*}=-(N-2)^{2} / 4$ and $\omega_{k} \geq k^{2}$, we see that

$$
N-3+\epsilon+2 A_{\lambda+\omega_{k}}^{+}=-1+\epsilon+\sqrt{(N-2)^{2}+4\left(\lambda+\omega_{k}\right)} \geq-1+\epsilon+2 k
$$

for $k \in\{0,1,2, \ldots\}$. It follows that

$$
\begin{aligned}
& s^{1-N}\left[v_{k, \lambda}^{+}(s)\right]^{-2} \int_{0}^{s} \tau^{N-1} v_{k, \lambda}^{+}(\tau)|f(\tau)| d \tau \\
& \leq s^{1-N-2 A_{\lambda+\omega_{k}}^{+}} \int_{0}^{s} \tau^{N-3+\epsilon+2 A_{\lambda+\omega_{k}}^{+}} d \tau \leq C(\epsilon+2 k)^{-1} s^{-1+\epsilon} \quad \text { for } \quad s \in(0, R] .
\end{aligned}
$$

Then we easily obtain (2.7) and (2.8), and the proof is complete.

Proof of Proposition 2.1. Let $V_{\lambda_{1}}(r):=V(r)-\lambda_{1} r^{-2}$ and $R \geq 1$. By condition (V) (ii) we find $C_{V}>0$ such that

$$
\left|V_{\lambda_{1}}(r)\right| \leq C_{V} r^{-2+\rho_{1}} \quad \text { for } \quad r>0 .
$$


Define $\left\{z_{n}\right\}_{n=1}^{\infty}$ inductively by

$$
z_{1}(r):=v_{k, \lambda_{1}}^{+}(r), \quad z_{n+1}(r):=v_{k, \lambda_{1}}^{+}(r)+F_{k, \lambda_{1}}^{+}\left[V_{\lambda_{1}} z_{n}\right](r), \quad n=1,2, \ldots
$$

We prove that there exists $C_{1}>0$, independent of $R$ and $k$, such that

$$
\left|z_{n+1}(r)-z_{n}(r)\right| \leq\left(\frac{C_{1} C_{V} R^{\rho_{1}}}{k+1}\right)^{n} v_{k, \lambda_{1}}^{+}(r), \quad r \in(0, R],
$$

for $n=1,2, \ldots$ By (2.9) we apply Lemma 2.1 to find $C_{*}>0$, independent of $k$, such that

$$
\left|z_{2}(r)-z_{1}(r)\right| \leq F_{k, \lambda_{1}}^{+}\left[\left|V_{\lambda_{1}}\right| v_{k, \lambda_{1}}^{+}\right](r) \leq \frac{C_{*} C_{V}}{k+1} R^{\rho_{1}} v_{k, \lambda_{1}}^{+}(r), \quad r \in(0, R],
$$

which implies (2.10) with $n=1$. If (2.10) holds for some $n_{*} \in\{1,2, \ldots\}$, then we apply Lemma 2.1 again to obtain

$$
\left|z_{n_{*}+2}(r)-z_{n_{*}+1}(r)\right| \leq F_{k, \lambda_{1}}^{+}\left[\left|V_{\lambda_{1}}\right|\left|z_{n_{*}+1}-z_{n_{*}}\right|\right](r) \leq\left(\frac{C_{*} C_{V} R^{\rho_{1}}}{k+1}\right)^{n_{*}+1} v_{k, \lambda_{1}}^{+}(r)
$$

for $r \in(0, R]$. Thus inequality (2.10) holds for $n=1,2, \ldots$ with $C_{1}=C_{*}$.

We prove assertion (a). Let $k \in\{0,1,2, \ldots\}$ and fix it. By the regularity of $h_{k}$, it suffices to treat the case when $R>0$ is small enough. By (2.10), taking small enough $R>0$, we have

$$
\left|z_{n+1}(r)-z_{n}(r)\right| \leq\left(\frac{1}{2}\right)^{n} v_{k, \lambda_{1}}^{+}(r), \quad r \in(0, R],
$$

for $n=1,2, \ldots$ Then, applying the standard theory for ordinary differential equations, we see that the limit function $z(r):=\lim _{n \rightarrow \infty} z_{n}(r)$ exists in $(0, R]$ and

$$
z(r)=v_{k, \lambda_{1}}^{+}(r)+F_{k, \lambda_{1}}^{+}\left[V_{\lambda_{1}} z\right](r), \quad|z(r)| \leq 2 v_{k, \lambda_{1}}^{+}(r),
$$

for $r \in(0, R]$. By Lemma 2.1 we find $C_{2}>0$, independent of $k$, such that

$$
\left|\frac{d^{\ell}}{d r^{\ell}} F_{k, \lambda_{1}}^{+}\left[V_{\lambda_{1}} z\right](r)\right| \leq C_{2}(k+1)^{\ell-1} r^{-\ell+\rho_{1}} v_{k, \lambda_{1}}^{+}(r)
$$

for $r \in(0, R]$, where $\ell \in\{0,1\}$. Furthermore, we see that $z$ is a solution to ODE (2.5) in $(0, R]$. Recalling that $h_{k}^{ \pm}$are linearly independent solutions to ODE (2.5), we find $a, b \in \mathbf{R}$ such that

$$
z(r)=a h_{k}^{+}(r)+b h_{k}^{-}(r) \quad \text { for } \quad r \in(0, R] .
$$

Since $h_{k}^{+}(r) / h_{k}^{-}(r) \rightarrow+0$ as $r \rightarrow+0$ and $z(r)=v_{k, \lambda_{1}}^{+}(r)(1+o(1))$ as $r \rightarrow+0$, we see that $a=1$ and $b=0$, that is, $z(r)=h_{k}^{+}(r)=h_{k}(r)$ for $r \in(0, R]$. This together with (2.12) implies assertion (a) for small enough $R>0$. Thus assertion (a) follows.

The proof of assertion (b) is similar. Let $R>0$ and fix it. By (2.10), taking large enough $k_{*} \in\{0,1,2, \ldots\}$, we have

$$
\left|z_{n+1}(r)-z_{n}(r)\right| \leq\left(\frac{1}{2}\right)^{n} v_{k, \lambda_{1}}^{+}(r), \quad r \in(0, R]
$$


for $n=1,2, \ldots$ and $k \geq k_{*}$. Similarly to assertion (a), we see that the limit function $z(r):=$ $\lim _{n \rightarrow \infty} z_{n}(r)$ exists in $(0, R]$ and $z$ satisfies (2.11), (2.12) and $z(r)=h_{k}(r)$ for $r \in(0, R]$ and $k \geq k_{*}$. Furthermore, by (2.11) and (2.12) we see that

$$
\left|\frac{d}{d r} h_{k}(r)-A_{\lambda_{1}+\omega_{k}}^{+} r^{-1} v_{k, \lambda_{1}}^{+}(r)\right| \leq C_{2} r^{-1+\rho_{1}} v_{k, \lambda_{1}}^{+}(r)
$$

for $r \in(0, R]$. Since $A_{\lambda_{1}+\omega_{k}}^{+}=k(1+o(1))$ as $k \rightarrow \infty$ (see (1.3)), taking large enough $k_{*}$ if necessary, we obtain

$$
\frac{d}{d r} h_{k}(r) \asymp A_{\lambda_{1}+\omega_{k}}^{+} r^{-1} v_{k, \lambda_{1}}^{+}(r) \asymp k r^{-1} v_{k, \lambda_{1}}^{+}(r)
$$

for $r \in(0, R]$ and large enough $k \geq k_{*}$. Then we complete the proof of assertion (b). Thus Proposition 2.1 follows.

Next we prove the following proposition on the behavior of $h_{k}$ as $r \rightarrow \infty$.

Proposition 2.2 Assume conditions (V) and (N'). Let $v_{k}$ be as in (1.5).

(a) Let $k \in\{0,1,2, \ldots\}$ and $\ell \in\{0,1\}$. Then

$$
\frac{d^{\ell}}{d r^{\ell}} h_{k}(r)=c_{k}(1+o(1)) \frac{d^{\ell}}{d r^{\ell}} v_{k}(r)+o\left(r^{-\ell} v_{k}(r)\right) \quad \text { as } \quad r \rightarrow \infty
$$

(b) There exist $C>0$ and $k_{*} \in\{0,1,2, \ldots\}$ such that

$$
C^{-1} \leq \frac{h_{k}(r)}{v_{k}(r)} \leq C \quad \text { in } \quad[1, \infty), \quad C^{-1} k r^{-1} v_{k}(r) \leq \frac{d}{d r} h_{k}(r) \leq C k r^{-1} v_{k}(r),
$$

for $r \geq 1$ and $k \in\left\{k_{*}, k_{*}+1, \ldots\right\}$.

Proof. Let $V_{\lambda_{2}}(r):=V(r)-\lambda_{2} r^{-2}, k \in\{0,1,2, \ldots\}$ and $\ell \in\{0,1\}$. We prove assertion (a).

Step 1: Assume either

$$
k \geq 1 \quad \text { or } \quad H \text { is subcritical and } \lambda>\lambda_{*} .
$$

It follows from (1.5) that $h_{k}(r)=c_{k} v_{k, \lambda_{2}}^{+}(r)(1+o(1))$ as $r \rightarrow \infty$. Let $R \geq 1$. Let $a_{k, R}, b_{k, R} \in \mathbf{R}$ be such that

$$
\begin{aligned}
& h_{k}(R)=a_{k, R} v_{k, \lambda_{2}}^{+}(R)+b_{k, R} v_{k, \lambda_{2}}^{-}(R), \\
& \frac{d}{d r} h_{k}(R)=a_{k, R} \frac{d}{d r} v_{k, \lambda_{2}}^{+}(R)+b_{k, R} \frac{d}{d r} v_{k, \lambda_{2}}^{-}(R) .
\end{aligned}
$$

Set $z_{0}(r):=a_{k, R} v_{k, \lambda_{2}}^{+}(r)+b_{k, R} v_{k, \lambda_{2}}^{-}(r)$ for $r \in(0, \infty)$. Then

$$
\left|z_{0}(r)\right| \leq m_{k, R} v_{k, \lambda_{2}}^{+}(r) \quad \text { for } \quad r \in[1, \infty) \text {, }
$$

where $m_{k, R}:=\left|a_{k, R}\right|+\left|b_{k, R}\right|$. Define $\left\{z_{n}\right\}_{n=0}^{\infty}$ inductively by

$$
z_{n+1}(r):=z_{0}(r)+G_{R}\left[z_{n}\right](r) \text { for } \quad r \in(0, \infty),
$$


where

$$
G_{R}\left[z_{n}\right](r):=v_{k, \lambda_{2}}^{-}(r) \int_{R}^{r} s^{1-N}\left[v_{k, \lambda_{2}}^{-}(s)\right]^{-2}\left(\int_{R}^{s} \tau^{N-1} v_{k, \lambda_{2}}^{-}(\tau) V_{\lambda_{2}}(\tau) z_{n}(\tau) d \tau\right) d s .
$$

On the other hand, by condition (V) (ii) we find $C_{V}^{\prime}>0$ such that

$$
\left|V_{\lambda_{2}}(r)\right| \leq C_{V}^{\prime} r^{-2-\rho_{2}} \quad \text { for } \quad r \in[1, \infty) .
$$

Then we have

$$
\left|z_{n+1}(r)-z_{n}(r)\right| \leq m_{k, R}\left(\frac{C_{V}^{\prime} I_{R}}{\sqrt{D_{k}}}\right)^{n+1} v_{k, \lambda_{2}}^{+}(r)
$$

for $r \in[R, \infty)$ and $k \in\{0,1,2, \ldots\}$, where

$$
I_{R}:=\int_{R}^{\infty} \tau^{-1-\rho_{2}} d \tau, \quad D_{k}:=D_{\lambda_{2}+\omega_{k}}=(N-2)^{2}+4\left(\lambda_{2}+\omega_{k}\right) \geq 4 k^{2} .
$$

Indeed, it follows from (2.15) that

$$
\int_{R}^{s} \tau^{N-1} v_{k, \lambda_{2}}^{-}(\tau)\left|V_{\lambda_{2}}(\tau)\right| v_{k, \lambda_{2}}^{+}(\tau) d \tau \leq C_{V}^{\prime} \int_{R}^{s} \tau^{-1-\rho_{2}} d \tau \leq C_{V}^{\prime} I_{R}
$$

for $s \in[R, \infty)$. This implies that

$$
\begin{aligned}
\left|G_{R}\left[v_{k, \lambda_{2}}^{+}\right](r)\right| & \leq C_{V}^{\prime} I_{R} v_{k, \lambda_{2}}^{-}(r) \int_{R}^{r} s^{1-N}\left[v_{k, \lambda_{2}}^{-}(s)\right]^{-2} d s \\
& =C_{V}^{\prime} I_{R} v_{k, \lambda_{2}}^{-}(r) \int_{R}^{r} s^{-1+\sqrt{D_{k}}} d s \leq \frac{C_{V}^{\prime} I_{R}}{\sqrt{D_{k}}} v_{k, \lambda_{2}}^{+}(r)
\end{aligned}
$$

for $r \in[R, \infty)$. This together with (2.14) implies (2.16) with $n=0$. Repeating this argument, by induction we see that (2.16) holds for $n=0,1,2, \ldots$.

Since $I_{R} \rightarrow 0$ as $R \rightarrow \infty$, we take large enough $R_{k} \geq 1$ so that

$$
\frac{C_{V}^{\prime} I_{R}}{\sqrt{D_{k}}} \leq \frac{1}{2} \quad \text { for } \quad R \geq R_{k}
$$

Then, for any $R \geq R_{k}$, applying the standard theory for ordinary differential equations, we see that $z(r):=\lim _{n \rightarrow \infty} z_{n}$ exists for $r \in[R, \infty)$ and $z$ satisfies

$$
z(r)=z_{0}(r)+G_{R}[z](r), \quad|z(r)| \leq 2 m_{k, R_{k}} v_{k, \lambda_{2}}^{+}(r),
$$

for $r \in[R, \infty)$. Furthermore,

$$
\begin{aligned}
& \frac{d^{2}}{d r^{2}} z+\frac{N-1}{r} \frac{d}{d r} z-\left(\lambda_{2}+\omega_{k}\right) r^{-2} z=V_{\lambda_{2}}(r) z \quad \text { in } \quad[R, \infty) \\
& z(R)=z_{0}(R)=h_{k}(R), \quad \frac{d}{d r} z(R)=\frac{d}{d r} z_{0}(R)=\frac{d}{d r} h_{k}(R) .
\end{aligned}
$$


On the other hand, by (1.4) we see that $h_{k}$ satisfies relation (2.20) with $z$ replaced by $h_{k}$. These imply that $h_{k}(r)=z(r)$ for $r \in[R, \infty)$. Therefore we obtain

$$
h_{k}(r)=a_{k, R} v_{k, \lambda_{2}}^{+}(r)+b_{k, R} v_{k, \lambda_{2}}^{-}(r)+G_{R}\left[h_{k}\right](r)
$$

for $r \geq R$, where $R \geq R_{k}$. On the other hand, under assumption (2.13), by (1.5) we have

$$
\left|h_{k}(r)\right| \leq C v_{k, \lambda_{2}}^{+}(r) \quad \text { for } \quad r \in[1, \infty) .
$$

Then, similarly to (2.17), we see that

$$
\left|\frac{d^{\ell}}{d r^{\ell}} G_{R}\left[h_{k}\right](r)\right| \leq C I_{R} r^{-\ell} v_{k, \lambda_{2}}^{+}(r)
$$

for $r \in[R, \infty)$. Since $h_{k}(r)=c_{k} v_{k, \lambda_{2}}^{+}(r)(1+o(1))$ as $r \rightarrow \infty$ (see (1.5)) and $I_{R} \rightarrow 0$ as $R \rightarrow \infty$, by (2.21) and (2.22) we see that $a_{k, R} \rightarrow c_{k}$ as $R \rightarrow \infty$ and obtain

$$
\frac{d^{\ell}}{d r^{\ell}} h_{k}(r)=c_{k}(1+o(1)) \frac{d^{\ell}}{d r^{\ell}} v_{k, \lambda_{2}}^{+}(r)+o\left(r^{-\ell} v_{k, \lambda_{2}}^{+}(r)\right) \quad \text { as } \quad r \rightarrow \infty .
$$

Thus assertion (a) follows under assumption (2.13).

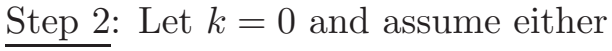

$$
\text { (i) } H \text { is subcritical and } \lambda_{2}=\lambda_{*} \quad \text { or } \quad \text { (ii) } H \text { is critical. }
$$

Set

$$
G(r):=v_{0, \lambda_{2}}^{+}(r) \int_{r}^{\infty} s^{1-N}\left[v_{0, \lambda_{2}}^{+}(s)\right]^{-2}\left(\int_{s}^{\infty} \tau^{N-1} v_{0, \lambda_{2}}^{+}(\tau) V_{\lambda_{2}}(\tau) h_{0}(\tau) d \tau\right) d s .
$$

It follows from (1.5) and (2.15) that

$$
\tau^{N-1} v_{0, \lambda_{2}}^{+}(\tau)\left|V_{\lambda_{2}}(\tau)\right| h_{0}(\tau) \leq C \tau^{N-1} \tau^{A_{\lambda_{2}}^{+}} \tau^{-2-\rho_{2}} \tau^{A_{\lambda_{2}}^{-}}(\log \tau)^{B_{0}}=C \tau^{-1-\rho_{2}}(\log \tau)^{B_{0}}
$$

for $\tau \geq 2$. Then we have

$$
|G(r)| \leq C v_{0, \lambda_{2}}^{+}(r) \int_{r}^{\infty} s^{-1-\sqrt{D_{0}}} s^{-\rho_{2}}(\log r)^{B_{0}} d s \leq C r^{-\rho_{2}} v_{0, \lambda_{2}}^{-}(r)
$$

for $r \geq 2$. This implies that $G(r)=o\left(h_{0}(r)\right)$ as $r \rightarrow \infty$. Furthermore, $G$ satisfies

$$
\frac{d^{2}}{d r^{2}} G+\frac{N-1}{r} \frac{d}{d r} G-\lambda_{2} r^{-2} G=V_{\lambda_{2}}(r) h_{0}(r) \quad \text { in } \quad(0, \infty) .
$$

Therefore, setting $\tilde{h}:=h_{0}-G$, we have

$$
\frac{d^{2}}{d r^{2}} \tilde{h}+\frac{N-1}{r} \frac{d}{d r} \tilde{h}-\lambda_{2} r^{-2} \tilde{h}=0 \quad \text { in } \quad(0, \infty)
$$

Since $v_{0, \lambda_{2}}^{ \pm}$are linearly independent solutions to ODE (2.26), we find $a, b \in \mathbf{R}$ such that

$$
h_{0}(r)-G(r)=\tilde{h}(r)=a v_{0, \lambda_{2}}^{+}(r)+b v_{0, \lambda_{2}}^{-}(r) \quad \text { for } \quad r>0 .
$$


On the other hand, it follows from (1.5) that $h_{0}(r)=c_{0} v_{0, \lambda_{2}}^{-}(r)(1+o(1))$ as $r \rightarrow \infty$. This implies that $a=0$ and $b=c_{0}$, that is,

$$
h_{0}(r)=c_{0} v_{0, \lambda_{2}}^{-}(r)+G(r) \quad \text { in } \quad(0, \infty) .
$$

Then assertion (a) easily follows from (2.24) and (2.25) under assumption (2.23). The proof of assertion (a) is complete.

Step 3: We prove assertion (b). In this step the letter $C$ denotes generic positive constants independent of $k$. Let $R=1$. Since $D_{k} \rightarrow \infty$ as $k \rightarrow \infty$, taking large enough $k_{*} \in\{1,2, \ldots\}$, we have

$$
\frac{C_{V}^{\prime} I_{1}}{\sqrt{D_{k}}} \leq C k^{-1} \leq \frac{1}{2} \quad \text { for } \quad k \geq k_{*},
$$

instead of (2.18). Then, similarly to (2.19) (with $R_{k}=1$ ), we see that $z(r):=\lim _{n \rightarrow \infty} z_{n}$ exists for $r \in[1, \infty)$ and $z$ satisfies

$$
z(r)=z_{0}(r)+G_{1}[z](r), \quad|z(r)| \leq 2 m_{k, 1} v_{k, \lambda_{2}}^{+}(r),
$$

for $r \in[1, \infty)$, where $k \geq k_{*}$. Furthermore, $z=h_{k}$ in $[1, \infty)$ and

$$
\begin{aligned}
& h_{k}(r)=a_{k, 1} v_{k, \lambda_{2}}^{+}(r)+b_{k, 1} v_{k, \lambda_{2}}^{-}(r)+G_{1}\left[h_{k}\right](r), \\
& \left|G_{1}\left[h_{k}\right](r)\right| \leq C k^{-1} v_{k, \lambda_{2}}^{+}(r), \quad\left|\frac{d}{d r} G_{1}\left[h_{k}\right](r)\right| \leq C r^{-1} v_{k, \lambda_{2}}^{+}(r),
\end{aligned}
$$

for $r \in[1, \infty)$. On the other hand, taking large enough $k_{*}$ if necessary, by Proposition 2.1 we see that

$$
C^{-1}<h_{k}(1) \leq C, \quad C^{-1} k \leq \frac{d}{d r} h_{k}(1) \leq C k,
$$

for $k \geq k_{*}$. These imply that

$$
C^{-1}<a_{k, 1}+b_{k, 1} \leq C, \quad C^{-1} k \leq A_{\lambda_{2}+\omega_{k}}^{+} a_{k, 1}+A_{\lambda_{2}+\omega_{k}}^{-} b_{k, 1} \leq C k,
$$

for $k \geq k_{*}$. Since

$$
D_{k}=(N-2)^{2}+4\left(\lambda_{2}+\omega_{k}\right)=4 k^{2}(1+o(1)) \quad \text { as } \quad k \rightarrow \infty,
$$

it follows from (2.27) that

$$
\begin{aligned}
k & \asymp A_{\lambda_{2}+\omega_{k}}^{+} a_{k, 1}+A_{\lambda_{2}+\omega_{k}}^{-} b_{k, 1}+\frac{\sqrt{D_{k}}}{2}\left(a_{k, 1}+b_{k, 1}\right) \\
& =\left(A_{\lambda_{2}+\omega_{k}}^{+}-A_{\lambda_{2}+\omega_{k}}^{-}\right) a_{k, 1}+A_{\lambda_{2}+\omega_{k}}^{-}\left(a_{k, 1}+b_{k, 1}\right)+\frac{\sqrt{D_{k}}}{2}\left(a_{k, 1}+b_{k, 1}\right) \\
& =\sqrt{D_{k}} a_{k, 1}-\frac{N-2}{2}\left(a_{k, 1}+b_{k, 1}\right)=2 k(1+o(1)) a_{k, 1}+O(1) \quad \text { as } \quad k \rightarrow \infty .
\end{aligned}
$$

This implies that

$$
C^{-1} \leq a_{k, 1} \leq C
$$


for large enough $k$. Then, by (2.27) we see that $\left|b_{k, 1}\right| \leq C$ for large enough $k$. Therefore we find $R \geq 1$ such that

$$
h_{k}(r) \asymp v_{k, \lambda_{2}}^{+}(r)=v_{k}(r)
$$

for $r \geq R$ and large enough $k$. Furthermore

$$
\begin{aligned}
& C^{-1} k r^{-1} v_{k, \lambda_{2}}^{+}(r)-C k r^{-1} v_{k, \lambda_{2}}^{-}(r) \\
& \leq \frac{d}{d r} h_{k}(r)=A_{\lambda_{2}+\omega_{k}}^{+} a_{k, 1} r^{-1} v_{k, \lambda_{2}}^{+}(r)+A_{\lambda_{2}+\omega_{k}}^{-} b_{k, 1} r^{-1} v_{k, \lambda_{2}}^{-}(r)+\frac{d}{d r} G_{1}\left[h_{k}\right](r) \\
& \leq C k r^{-1} v_{k, \lambda_{2}}^{+}(r)+C k r^{-1} v_{k, \lambda_{2}}^{-}(r)
\end{aligned}
$$

for $r \geq 1$ and large enough $k$. Taking large enough $R$ if necessary, we see that

$$
\frac{1}{2} C^{-1} k r^{-1} v_{k, \lambda_{2}}^{+}(r) \leq \frac{d}{d r} h_{k}(r) \leq 2 C k r^{-1} v_{k, \lambda_{2}}^{+}(r)
$$

for $r \in[R, \infty)$ and large enough $k$. Combining Proposition 2.1 (b) with (2.28) and (2.29), we complete the proof of assertion (b). Thus Proposition 2.2 follows.

Combining Propositions 2.1 and 2.2, we have:

Proposition 2.3 Assume conditions (V) and (N'). Then there exists $C>0$ such that

$$
C^{-1} \leq \frac{h_{k}(r)}{v_{k, \lambda_{1}}^{+}(r)} \leq C \quad \text { in } \quad(0,1], \quad C^{-1} \leq \frac{h_{k}(r)}{v_{k}(r)} \leq C \quad \text { in } \quad(1, \infty),
$$

for $k \in\{0,1,2, \ldots\}$.

At the end of this subsection we prove the following proposition.

Proposition 2.4 Assume conditions (V) and (N').

(a) Let $(p, p, \sigma, \sigma) \in \Lambda$ be such that $h_{0} \in L^{p, \sigma}(B(0,1))$. Then there exists $C_{1}>0$ such that

$$
\frac{\left\|h_{0}\right\|_{L^{p, \sigma}(B(0, \sqrt{t}))}}{h_{0}(\sqrt{t})} \geq C_{1} t^{\frac{N}{2 p}} \quad \text { for } \quad t>0 .
$$

(b) There exists $C_{2}>0$ such that

$$
\int_{0}^{r} s^{N-1} h_{k}(s)^{2} d s \leq C_{2}(k+1)^{-1} r^{N} h_{k}(r)^{2}
$$

for $r>0$ and $k \in\{0,1,2, \ldots\}$.

Proof. By (1.4) and (1.5) we find $C>0$ and $\epsilon \in(0,1)$ such that

$$
\frac{\left\|h_{0}^{\epsilon}\right\|_{L^{1}(B(0, \sqrt{t}))}}{h_{0}(\sqrt{t})^{\epsilon}} \geq C t^{\frac{N}{2}} \quad \text { for } \quad t>0 .
$$

It follows from (2.1) that

$$
\left\|h_{0}^{\epsilon}\right\|_{L^{1}(B(0, \sqrt{t}))} \leq|B(0, \sqrt{t})|^{1-\frac{\epsilon}{p}}\left\|h_{0}\right\|_{L^{p, \sigma}(B(0, \sqrt{t}))}^{\epsilon} \quad \text { for } \quad t>0 .
$$

These imply assertion (a). Assertion (b) follows from Proposition 2.3 (see also [18, (3.7)]). 


\section{$3 L^{p, \sigma}-L^{q, \theta}$ estimates of $e^{-t H}$}

The aim of this section is to prove the following proposition.

Proposition 3.1 Assume conditions (V) and ( $\left.\mathrm{N}^{\prime}\right)$. Let $(p, q, \sigma, \theta) \in \Lambda$.

(a) Let $\ell \in\{0,1\}, j \in\{0,1,2, \ldots\}$ and $\delta \in(0,1]$. Then there exist $C_{1}>0$ and $C_{2}>0$ such that

$$
\begin{aligned}
\left\|\partial_{t}^{j} \nabla^{\ell} e^{-t H} \phi\right\|_{L^{q, \theta}\left(B(0, \delta \sqrt{t})^{c}\right)} & \leq C_{1} t^{-\frac{N}{2}\left(1-\frac{1}{q}\right)-\frac{\ell}{2}-j}\left[\frac{\left\|h_{0} \phi\right\|_{L^{1}(B(0, \sqrt{t}))}}{h_{0}(\sqrt{t})}+t^{\frac{N}{2 p^{\prime}}}\|\phi\|_{L^{p, \sigma}\left(B(0, \delta \sqrt{t})^{c}\right)}\right] \\
& \leq C_{2} t^{-\frac{N}{2}\left(1-\frac{1}{q}\right)-\frac{\ell}{2}-j} \frac{\left\|h_{0}\right\|_{L^{p^{\prime}, \sigma^{\prime}}(B(0, \sqrt{t}))}}{h_{0}(\sqrt{t})}\|\phi\|_{L^{p, \sigma}}
\end{aligned}
$$

for $\phi \in C_{c}\left(\mathbf{R}^{N}\right)$ and $t>0$.

(b) There exist $C_{3}>0$ and $C_{4}>0$ such that

$$
\begin{aligned}
\left\|e^{-t H} \phi\right\|_{L^{q, \theta}} & \leq C_{3} t^{-\frac{N}{2}} \frac{\left\|h_{0}\right\|_{L^{q, \theta}(B(0, \sqrt{t}))}}{h_{0}(\sqrt{t})}\left[\frac{\left\|h_{0} \phi\right\|_{L^{1}(B(0, \sqrt{t}))}}{h_{0}(\sqrt{t})}+t^{\frac{N}{2 p^{\prime}}}\|\phi\|_{L^{p, \sigma}\left(B(0, \sqrt{t})^{c}\right)}\right] \\
& \leq C_{4} t^{-\frac{N}{2}} \frac{\left\|h_{0}\right\|_{L^{q, \theta}(B(0, \sqrt{t}))}\left\|h_{0}\right\|_{L^{p^{\prime}, \sigma^{\prime}}(B(0, \sqrt{t}))}}{h_{0}(\sqrt{t})^{2}}\|\phi\|_{L^{p, \sigma}}
\end{aligned}
$$

for $\phi \in C_{c}\left(\mathbf{R}^{N}\right)$ and $t>0$.

We first recall the following lemma on an upper Gaussian estimate of $p=p(x, y, t)$. See [17, Theorem 1.3].

Lemma 3.1 Assume conditions (V) and (N'). Then there exists $C>0$ such that

$$
0<p(x, y, t) \leq C t^{-\frac{N}{2}} \frac{\tilde{h}_{0}(x, t) \tilde{h}_{0}(y, t)}{h_{0}(\sqrt{t})^{2}} \exp \left(-\frac{|x-y|^{2}}{C t}\right)
$$

for $x, y \in \mathbf{R}^{N} \backslash\{0\}$ and $t>0$, where $\tilde{h}_{0}(x, t):=h_{0}(\min \{|x|, \sqrt{t}\})$.

Combining Lemma 3.1 and the parabolic regularity theorems, we have:

Lemma 3.2 Assume conditions (V) and (N'). Let $\ell \in\{0,1\}, j \in\{0,1,2, \ldots\}$ and $\delta \in(0,1]$. Then there exists $C>0$ such that

$$
\left|\partial_{t}^{j} \nabla^{\ell} p(x, y, t)\right| \leq C t^{-\frac{N}{2}-\frac{\ell}{2}-j} \frac{\tilde{h}_{0}(x, t) \tilde{h}_{0}(y, t)}{h_{0}(\sqrt{t})^{2}} \exp \left(-\frac{|x-y|^{2}}{C t}\right)
$$

for $x \in B(0, \delta \sqrt{t})^{c}, y \in \mathbf{R}^{N} \backslash\{0\}$ and $t>0$. 
Proof. Let $y \in \mathbf{R}^{N} \backslash\{0\}, t>0$ and $\delta \in(0,1]$. Set $k=\delta \sqrt{t} / 2$. Let $x \in B(0, \delta \sqrt{t})^{c}$ and set $p_{k}(z, s):=p\left(x+k z, y, t+k^{2} s\right)$ for $(z, s) \in B(0,1) \times(-1,1)$. Then $p_{k}$ satisfies

$$
\partial_{s} p_{k}-\Delta_{z} p_{k}+\tilde{V}(z) p_{k}=0 \quad \text { in } \quad(x, t) \in B(0,1) \times(-1,1),
$$

where $\tilde{V}(z)=k^{2} V(|x+k z|)$. By condition (V) (iii) we have

$$
|\nabla \tilde{V}(z)| \leq C k^{3}|x+k z|^{-3} \leq C \quad \text { for } \quad z \in B(0,1) .
$$

Let $\ell \in\{0,1\}$ and $j \in\{0,1,2, \ldots\}$. By (3.2) we apply the parabolic regularity theorems to (3.1) and obtain

$$
k^{\ell+2 j}\left|\partial_{t}^{j} \nabla^{\ell} p(x, y, t)\right|=\left|\partial_{t}^{j} \nabla^{\ell} p_{k}(0,0)\right| \leq C\left\|p_{k}\right\|_{L^{\infty}(B(0,1) \times(-1,1))} .
$$

Then, due to the relation that $h_{0}(r / 2) \asymp h_{0}(r) \asymp h_{0}(2 r)$ for $r>0$ (see (1.4) and (1.5)), by Lemma 3.1 we obtain the desired inequality.

We prove Proposition 3.1 by using Lemmas 3.1 and 3.2 .

Proof of Proposition 3.1. Let $(p, q, \sigma, \theta) \in \Lambda$ and $\phi \in L^{p, \sigma}$. By Lemmas 3.1 and 3.2 we find $c>0$ such that

$$
\left|\left[\partial_{t}^{j} \nabla^{\ell} e^{-t H} \phi\right](x)\right| \leq C t^{-\frac{\ell}{2}-j}[I(x, t)+J(x, t)], \quad t>0,
$$

for $x \in B(0, \delta \sqrt{t})^{c}$ if $(j, \ell) \neq(0,0)$ and $x \in \mathbf{R}^{N} \backslash\{0\}$ if $(j, \ell)=(0,0)$, where

$$
\begin{aligned}
& I(x, t):=h_{0}(\sqrt{t})^{-2} \tilde{h}_{0}(x, t) \int_{B(0, \sqrt{t})} h_{0}(|y|) G_{c}(x-y, t)|\phi(y)| d y, \\
& J(x, t):=h_{0}(\sqrt{t})^{-1} \tilde{h}_{0}(x, t) \int_{B(0, \sqrt{t})^{c}} G_{c}(x-y, t)|\phi(y)| d y \\
& G_{c}(x, t):=t^{-\frac{N}{2}} \exp \left(-\frac{|x|^{2}}{c t}\right) .
\end{aligned}
$$

We prove assertion (a). Let $\delta \in(0,1], x \in B(0, \delta \sqrt{t})^{c}$ and $t>0$. Thanks to (1.4) and (1.5), we have $\tilde{h}_{0}(x, t) \leq C h_{0}(\sqrt{t})$. Then it follows from (2.2) that

$$
\begin{aligned}
\|I(t)\|_{L^{q, \theta}\left(B(0, \delta \sqrt{t})^{c}\right)} & \leq C h_{0}(\sqrt{t})^{-1}\left\|\int_{\mathbf{R}^{N}} G_{c}(\cdot-y, t) h_{0}(|y|)|\phi(y)| \chi_{B(0, \sqrt{t})}(y) d y\right\|_{L^{q, \theta}} \\
& \leq C h_{0}(\sqrt{t})^{-1}\left\|G_{c}(t)\right\|_{L^{q, \theta}}\left\|h_{0} \phi\right\|_{L^{1}(B(0, \sqrt{t}))} \\
& \leq C t^{-\frac{N}{2}\left(1-\frac{1}{q}\right)} h_{0}(\sqrt{t})^{-1}\left\|h_{0} \phi\right\|_{L^{1}(B(0, \sqrt{t}))} .
\end{aligned}
$$

Similarly, by (2.2) we obtain

$$
\begin{aligned}
\|J(t)\|_{L^{q, \theta}\left(B(0, \delta \sqrt{t})^{c}\right)} & \leq\left\|G_{c}(t)\right\|_{L^{r, s}}\|\phi\|_{L^{p, \sigma}\left(B(0, \delta \sqrt{t})^{c}\right)} \\
& \leq C t^{-\frac{N}{2}\left(1-\frac{1}{r}\right)}\|\phi\|_{L^{p, \sigma}\left(B(0, \delta \sqrt{t})^{c}\right)}=C t^{-\frac{N}{2}\left(\frac{1}{p}-\frac{1}{q}\right)}\|\phi\|_{L^{p, \sigma}\left(B(0, \delta \sqrt{t})^{c}\right)}
\end{aligned}
$$


for $t>0$, where $1 \leq r \leq \infty$ and $1 \leq s \leq \infty$ with

$$
\frac{1}{r}+\frac{1}{p}=\frac{1}{q}+1, \quad \frac{1}{\theta}=\frac{1}{s}+\frac{1}{\sigma} .
$$

Combining (3.3), (3.4) and (3.5), we see that

$$
\left\|\partial_{t}^{j} \nabla^{\ell} e^{-t H} \phi\right\|_{L^{q, \theta}\left(B(0, \delta \sqrt{t})^{c}\right)} \leq C t^{-\frac{N}{2}\left(1-\frac{1}{q}\right)-\frac{\ell}{2}-j}\left[\frac{\left\|h_{0} \phi\right\|_{L^{1}(B(0, \sqrt{t}))}}{h_{0}(\sqrt{t})}+t^{\frac{N}{2 p^{\prime}}}\|\phi\|_{L^{p, \sigma}\left(B(0, \delta \sqrt{t})^{c}\right)}\right],
$$

which together with Proposition 2.4 (a) and (2.1) implies assertion (a).

We prove assertion (b). Let $x \in B(0, \sqrt{t})$ and $t>0$. It follows from $\tilde{h}_{0}(x, t)=h_{0}(|x|)$ that

$$
I(x, t) \leq h_{0}(\sqrt{t})^{-2} h_{0}(|x|) \int_{B(0, \sqrt{t})} h_{0}(|y|) G_{c}(x-y, t)|\phi(y)| d y,
$$

which implies that

$$
\begin{aligned}
\|I(t)\|_{L^{q, \theta}(B(0, \sqrt{t}))} & \leq C \frac{\left\|h_{0}\right\|_{L^{q, \theta}(B(0, \sqrt{t}))}}{h_{0}(\sqrt{t})^{2}} \sup _{x \in B(0, \sqrt{t})} \int_{B(0, \sqrt{t})} h_{0}(|y|) G_{c}(x-y, t)|\phi(y)| d y \\
& \leq C \frac{\left\|h_{0}\right\|_{L^{q, \theta}(B(0, \sqrt{t}))}}{h_{0}(\sqrt{t})^{2}} \cdot C t^{-\frac{N}{2}}\left\|h_{0} \phi\right\|_{L^{1}(B(0, \sqrt{t}))}
\end{aligned}
$$

for $t>0$. Similarly, we obtain

$$
\begin{aligned}
\|J(t)\|_{L^{q, \theta}(B(0, \sqrt{t}))} & \leq C h_{0}(\sqrt{t})^{-1}\left\|h_{0}\right\|_{L^{q, \theta}(B(0, \sqrt{t}))} \sup _{x \in B(0, \sqrt{t})} \int_{B(0, \sqrt{t})^{c}} G_{c}(x-y, t)|\phi(y)| d y \\
& \leq C t^{-\frac{N}{2 p}} \frac{\left\|h_{0}\right\|_{L^{q, \theta}(B(0, \sqrt{t}))}}{h_{0}(\sqrt{t})}\|\phi\|_{L^{p, \sigma}\left(B(0, \sqrt{t})^{c}\right)}
\end{aligned}
$$

for $t>0$. By assertion (a) with $(j, \ell)=(0,0)$, (3.6) and (3.7) we have

$$
\left\|e^{-t H} \phi\right\|_{L^{q, \theta}} \leq C t^{-\frac{N}{2}}\left[\frac{\left\|h_{0}\right\|_{L^{q, \theta}(B(0, \sqrt{t}))}}{h_{0}(\sqrt{t})}+t^{\frac{N}{2 q}}\right]\left[\frac{\left\|h_{0} \phi\right\|_{L^{1}(B(0, \sqrt{t}))}}{h_{0}(\sqrt{t})}+t^{\frac{N}{2 p^{\prime}}}\|\phi\|_{L^{p, \sigma}\left(B(0, \sqrt{t})^{c}\right)}\right]
$$

for $t>0$. This together with Proposition 2.4 (a) and (2.1) implies assertion (b). Thus Proposition 3.1 follows.

\section{Radially symmetric solutions}

In this section we obtain uniform estimates of time derivatives and spatial derivatives of $v_{k, i}$ inside parabolic cones with respect to $k$ and $i$. 


\subsection{Estimates of time derivatives}

Let $j \in\{0,1,2, \ldots\}$. In this subsection we prove the following proposition on uniform estimates of $\partial_{t}^{j} v_{k, i}$. Proposition 4.1 is one of the main ingredients in the proof of Theorem [1.1.

Proposition 4.1 Assume conditions (V) and ( $\left.\mathrm{N}^{\prime}\right)$. Let $(p, p, \sigma, \sigma) \in \Lambda$ be such that $h_{0} \in$ $L^{p, \sigma}(B(0,1)) \cap L^{p^{\prime}, \sigma^{\prime}}(B(0,1))$. Let $v_{k, i}$ be as in (1.9). Then, for any $j \in\{0,1,2, \ldots\}$, there exist $C>0$ and $\delta \in(0,1]$ such that

$$
\left|\partial_{t}^{j} v_{k, i}(x, t)\right| \leq C M_{k, i} t^{-\frac{N}{2}-j} \frac{\left\|h_{0}\right\|_{L^{p^{\prime}, \sigma^{\prime}}(B(0, \sqrt{t}))}}{h_{k}(\delta \sqrt{t}) h_{0}(\sqrt{t})} h_{k}(|x|)\|\phi\|_{L^{p, \sigma}}
$$

for $x \in B(0, \delta \sqrt{t}), t>0, k \in\{0,1,2, \ldots\}$ and $i \in\left\{1, \ldots, d_{k}\right\}$. Here $M_{k, i}:=\left\|Q_{k, i}\right\|_{L^{\infty}\left(\mathbf{S}^{N-1}\right)}$.

For the proof of Proposition 4.1, we start by proving the following lemma.

Lemma 4.1 Assume the same conditions as in Proposition 4.1. Then, for any $\delta \in(0,1]$ and $j \in\{0,1,2, \ldots\}$, there exists $C>0$ such that

$$
\left|\partial_{t}^{j} v_{k, i}\left(x, t+t_{0}\right)\right| \leq C M_{k, i} t^{-\frac{N}{2}-j} \frac{\left\|h_{0}\right\|_{L^{p^{\prime}, \sigma^{\prime}}(B(0, \sqrt{t}))}}{h_{0}(\sqrt{t})}\|\phi\|_{L^{p, \sigma}}
$$

for $x \in \partial B(0, \delta \sqrt{t}), t>0, t_{0} \in[0,1], k \in\{0,1,2, \ldots\}$ and $i \in\left\{1, \ldots, d_{k}\right\}$.

Proof. Let $\phi \in C_{c}\left(\mathbf{R}^{N}\right), j \in\{0,1,2, \ldots\}$ and $\delta \in(0,1]$. Let $u(t):=e^{-t H} \phi$ for $t>0$. By Proposition 3.1 (a) with $q=\theta=\infty$, we have

$$
\left|\partial_{t}^{j} u\left(x, t+t_{0}\right)\right| \leq C t^{-\frac{N}{2}-j} \frac{\left\|h_{0}\right\|_{L^{p^{\prime}, \sigma^{\prime}}(B(0, \sqrt{t}))}}{h_{0}(\sqrt{t})}\left\|u\left(t_{0}\right)\right\|_{L^{p, \sigma}}
$$

for $x \in \partial B(0, \delta \sqrt{t}), t>0$ and $t_{0} \in[0,1]$. Since $h_{0} \in L^{p, \sigma}(B(0,1)) \cap L^{p^{\prime}, \sigma^{\prime}}(B(0,1))$, by Proposition 3.1 (b) and (2.4) we have

$$
\left\|u\left(t_{0}\right)\right\|_{L^{p, \sigma}} \leq C t_{0}^{-\frac{N}{2}} \frac{\left\|h_{0}\right\|_{L^{p, \sigma}\left(B\left(0, \sqrt{t_{0}}\right)\right.}\left\|h_{0}\right\|_{L^{p^{\prime}, \sigma^{\prime}}\left(B\left(0, \sqrt{t_{0}}\right)\right)}}{h_{0}\left(\sqrt{t_{0}}\right)^{2}}\|\phi\|_{L^{p, \sigma}} \leq C\|\phi\|_{L^{p, \sigma}}
$$

for $t_{0} \in(0,1]$. These imply that

$$
\left|\partial_{t}^{j} u\left(x, t+t_{0}\right)\right| \leq C t^{-\frac{N}{2}-j} \frac{\left\|h_{0}\right\|_{L^{p^{\prime}, \sigma^{\prime}}(B(0, \sqrt{t}))}}{h_{0}(\sqrt{t})}\|\phi\|_{L^{p, \sigma}}
$$

for $x \in \partial B(0, \delta \sqrt{t}), t>0$ and $t_{0} \in[0,1]$. On the other hand, it follows from (1.10) that

$$
\int_{\partial B(0,1)} \partial_{t}^{j} u(|x| y, t) Q_{k, i}(y) d \sigma_{y}=\partial_{t}^{j} v_{k, i}(|x|, t)
$$

for $x \in \mathbf{R}^{N} \backslash\{0\}$ and $t>0$. This together with (4.3) implies (4.2). Thus Lemma 4.1 follows.

Next we prepare the following lemma on supersolutions. 
Lemma 4.2 Assume the same conditions as in Proposition 4.1. Let $k \in\{0,1,2, \ldots\}, T \geq 0$ and $M>0$. Let $\zeta \in C^{1}((T, \infty))$ be such that

$$
0 \leq-\zeta^{\prime}(t) \leq(k+1) M t^{-1} \zeta(t) \text { in } \quad(T, \infty)
$$

Set

$$
\begin{aligned}
f_{k}(|x|) & :=\int_{0}^{|x|} s^{1-N} \nu_{k}(s)^{-1}\left(\int_{0}^{s} \tau^{N-1} \nu_{k}(\tau) d \tau\right) d s, \quad \nu_{k}(|x|)=h_{k}(|x|)^{2}, \\
z_{*}(x, t) & :=2 \zeta(t)\left[1-(k+1) M t^{-1} f_{k}(|x|)\right],
\end{aligned}
$$

for $(x, t) \in \mathbf{R}^{N} \times(T, \infty)$. Then $z_{*}$ satisfies

$$
\partial_{t} z_{*} \geq \frac{1}{\nu_{k}} \operatorname{div}\left(\nu_{k} \nabla z_{*}\right), \quad z_{*} \leq 2 \zeta(t)
$$

for $(x, t) \in \mathbf{R}^{N} \times(T, \infty)$. Furthermore, there exists $\delta>0$ such that

$$
0 \leq(k+1) M t^{-1} f_{k}(|x|) \leq \frac{1}{2}, \quad z_{*}(x, t) \geq \zeta(t),
$$

for $x \in B(0, \delta \sqrt{t})$ and $t>T$. Here the constant $\delta$ depends on $M$ and it is independent of $k$.

Proof. We see that $f_{k}$ is nonnegative and it satisfies

$$
\frac{1}{\nu_{k}} \operatorname{div}\left(\nu_{k} \nabla f_{k}\right)=1 \quad \text { in } \quad \mathbf{R}^{N}
$$

Furthermore, $\zeta^{\prime} \leq 0$ in $(T, \infty)$. Then we observe that $z_{*}(x, t) \leq 2 \zeta(t)$ and

$$
\begin{aligned}
& \partial_{t} z_{*}-\frac{1}{\nu_{k}} \operatorname{div}\left(\nu_{k} \nabla z_{*}\right) \\
& =2 \zeta^{\prime}(t)\left[1-(k+1) M t^{-1} f_{k}(x)\right]+2(k+1) M \zeta(t) t^{-2} f_{k}(x)+2(k+1) M t^{-1} \zeta(t) \\
& \geq 2 \zeta^{\prime}(t)+2(k+1) M t^{-1} \zeta(t) \geq 0
\end{aligned}
$$

for $(x, t) \in \mathbf{R}^{N} \times(T, \infty)$. Furthermore, by Proposition 2.4 (b) we find $C>0$, independent of $k$, such that

$$
M(k+1) f_{k}(x) \leq C M \int_{0}^{|x|} s d s=\frac{C M}{2}|x|^{2} \leq \frac{C M}{2} \delta^{2} t
$$

for $x \in B(0, \delta \sqrt{t})$ and $t>T$. Then, taking a sufficiently small $\delta \in(0,1]$, we see that $z_{*}$ satisfies (4.4). Thus Lemma 4.2 follows.

We are ready to prove Proposition 4.1,

Proof of Proposition 4.1. The proof is divided into three steps. Let $j \in\{0,1,2, \ldots\}$ and fix it.

Step 1: We prove inequality (4.1) for $0<t \leq 1$. In this step the letter $C$ denotes generic positive constants independent of $k$. Let $\epsilon \in(0,1)$. Set

$$
\tilde{v}_{j}(x, t):=\partial_{t}^{j} v_{k, i}(x, t), \quad \tilde{w}_{j}(x, t):=\frac{\tilde{v}_{j}(x, t)}{h_{k}(|x|)}, \quad \eta_{j}(t):=t^{-\frac{N}{2 p}-j-\frac{A_{1, k}}{2}}
$$


for $(x, t) \in \mathbf{R}^{N} \times(0, \infty)$. Then $\tilde{w}_{j}$ satisfies

$$
\partial_{t} w=\frac{1}{\nu_{k}} \operatorname{div}\left(\nu_{k} \nabla w\right) \quad \text { in } \quad \mathbf{R}^{N} \times(0, \infty) .
$$

(See also Definition [2.1.) Since $\tilde{w}_{j}(\cdot, t)$ is radially symmetric for any $t>0$, it follows from [18, Lemma 2.2] that $\tilde{w}_{j} \in C\left(\mathbf{R}^{N} \times(0, \infty)\right)$. On the other hand, it follows from $h_{0} \in L^{p, \sigma}(B(0,1))$, (1.3) and (1.4) that $N+p A_{1,0} \geq 0$ and

$$
A_{1, k}=\sqrt{\omega_{k}}(1+o(1))=k(1+o(1)) \quad \text { as } \quad k \rightarrow \infty .
$$

Then we find $M_{1}>0$ such that

$$
-M_{1}(k+1) \leq-\frac{N}{2 p}-j-\frac{A_{1, k}}{2}-\epsilon \leq-\frac{1}{2 p}\left(N+p A_{1,0}\right)-\epsilon<0
$$

for $k \in\{0,1,2, \ldots\}$. This implies that

$$
0 \leq-\frac{t}{t^{-\epsilon} \eta_{j}(t)} \frac{d}{d t}\left(t^{-\epsilon} \eta_{j}(t)\right) \leq M_{1}(k+1) \quad \text { for } \quad t>0 .
$$

Let $z_{*}$ and $\delta \in(0,1]$ be as in Lemma 4.2 with $\zeta(t)$ and $T$ replaced by $t^{-\epsilon} \eta_{j}(t)$ and 0 , respectively. Here $\delta$ depends only on $M_{1}$ and it is independent of $k$ and $\epsilon$.

On the other hand, by Proposition 2.3 we see that

$$
h_{k}(|x|) \asymp|x|^{A_{1, k}} \quad \text { for } x \in B(0,1) \text { and } k \in\{0,1,2, \ldots\} .
$$

Then, by (2.4) we have

$$
C^{-1} \eta_{j}(t) \leq t^{-\frac{N}{2}-j} \frac{\left\|h_{0}\right\|_{L^{p^{\prime}, \sigma^{\prime}}(B(0, \sqrt{t}))}}{h_{k}(\delta \sqrt{t}) h_{0}(\sqrt{t})} \leq C \eta_{j}(t) \quad \text { for } \quad t \in(0,1]
$$

This together with Lemmas 4.1 and 4.2 implies that

$$
\begin{aligned}
\left|\tilde{w}_{j}\left(x, t+t_{0}\right)\right| & \leq C M_{k, i} t^{-\frac{N}{2}-j} \frac{\left\|h_{0}\right\|_{L^{p^{\prime}, \sigma^{\prime}}(B(0, \sqrt{t}))}}{h_{k}(\delta \sqrt{t}) h_{0}(\sqrt{t})}\|\phi\|_{L^{p, \sigma}} \\
& \leq C M_{k, i} t^{-\epsilon} \eta_{j}(t)\|\phi\|_{L^{p, \sigma}} \leq C M_{k, i}\|\phi\|_{L^{p, \sigma} z_{*}}(x, t)
\end{aligned}
$$

for $x \in \partial B(0, \delta \sqrt{t}), t \in(0,1], t_{0} \in(0,1]$ and $k \in\{0,1,2, \ldots\}$. Since $\tilde{w}_{j} \in C\left(\mathbf{R}^{N} \times[0, \infty)\right)$ and

$$
\inf _{x \in B(0, \delta \sqrt{t})} z_{*}(x, t) \geq t^{-\epsilon} \eta_{j}(t) \rightarrow \infty \quad \text { as } \quad t \rightarrow+0
$$

we see that

$$
\tilde{w}_{j}\left(x, t_{*}+t_{0}\right) \leq C M_{k, i}\|\phi\|_{L^{p, \sigma}} z_{*}\left(x, t_{*}\right)
$$

for $x \in B(0, \delta \sqrt{t})$ and small enough $t_{*}>0$. By Lemma 4.2, (4.5), (4.6) and (4.7) we apply the comparison principle to obtain

$$
\begin{aligned}
\left|\tilde{w}_{j}\left(x, t+t_{0}\right)\right| & \leq C M_{k, i}\|\phi\|_{L^{p, \sigma} z_{*}}(x, t) \leq C M_{k, i}\|\phi\|_{L^{p, \sigma}} t^{-\epsilon} \eta_{j}(t) \\
& \leq C M_{k, i} t^{-\frac{N}{2}-j-\epsilon} \frac{\left\|h_{0}\right\|_{L^{p^{\prime}, \sigma^{\prime}}(B(0, \sqrt{t}))}}{h_{k}(\delta \sqrt{t}) h_{0}(\sqrt{t})}\|\phi\|_{L^{p, \sigma}}
\end{aligned}
$$


for $x \in B(0, \delta \sqrt{t})$ and $t \in\left[t_{*}, 1\right]$. Letting $t_{*} \rightarrow+0$, we see that (4.8) holds for $x \in B(0, \delta \sqrt{t})$ and $t \in(0,1]$. Since $\epsilon \in(0,1)$ and $t_{0} \in(0,1)$ are arbitrary, we obtain inequality (4.1) for $x \in B(0, \delta \sqrt{t}), 0<t \leq 1$ and $k \in\{0,1,2, \ldots\}$. It remains to prove inequality (4.1) for $t>1$.

Step 2: Let $k \in\{0,1,2, \ldots\}$ and fix it. We prove that inequality (4.11) holds for $t>1$. In this step the letter $C$ denotes generic positive constants depending on $k$ possibly. By (4.8) we have

$$
\left|\tilde{w}_{j}(x, 1)\right| \leq C M_{k, i}\|\phi\|_{L^{p, \sigma}} \quad \text { for } \quad x \in B(0, \delta) .
$$

Furthermore, by Lemma 4.1 we see that

$$
\left|\tilde{w}_{j}(x, t)\right| \leq C M_{k, i} t^{-\frac{N}{2}-j} \frac{\left\|h_{0}\right\|_{L^{p^{\prime}, \sigma^{\prime}}(B(0, \sqrt{t}))}}{h_{k}(\delta \sqrt{t}) h_{0}(\sqrt{t})}\|\phi\|_{L^{p, \sigma}}
$$

for $x \in \partial B(0, \delta \sqrt{t})$ and $t \geq 1$. On the other hand, by (1.5) we find $\alpha \in \mathbf{R}$ and $\beta \in \mathbf{R}$ such that

$$
t^{-\frac{N}{2}-j} \frac{\left\|h_{0}\right\|_{L^{p^{\prime}, \sigma^{\prime}}(B(0, \sqrt{t}))}}{h_{k}(\delta \sqrt{t}) h_{0}(\sqrt{t})} \asymp t^{-\alpha}[\log (1+t)]^{\beta} \quad \text { for } \quad t \geq 1 .
$$

Assume either $\alpha>0$ or $\alpha=0, \beta<0$. Then we find $L \geq 1$ such that

$$
\eta_{2}(t):=t^{-\alpha}[\log (L+t)]^{\beta}
$$

is monotone decreasing in $(1, \infty)$. Furthermore, we also find $M_{2}>0$ such that

$$
0 \leq-\frac{t \eta_{2}^{\prime}(t)}{\eta_{2}(t)} \leq M_{2} \quad \text { in } \quad(1, \infty)
$$

By Lemma 4.2 we find a supersolution $z_{*}$ to problem $\left(\mathrm{W}_{k}\right)$ and $\delta_{k} \in(0, \delta)$ such that

$$
\begin{array}{ll}
z_{*} \leq 2 \eta_{2}(t) & \text { for }(x, t) \in \mathbf{R}^{N} \times(1, \infty), \\
z_{*}(x, t) \geq \eta_{2}(t) & \text { for }(x, t) \in B\left(0, \delta_{k} \sqrt{t}\right) \times(1, \infty) .
\end{array}
$$

Furthermore, by (4.9) we have

$$
\left|\tilde{w}_{j}(x, 1)\right| \leq C M_{k, i}\|\phi\|_{L^{p, \sigma}}=\frac{C M_{k, i}}{\eta_{2}(1)}\|\phi\|_{L^{p, \sigma}} \eta_{2}(1) \leq \frac{C M_{k, i}}{\eta_{2}(1)}\|\phi\|_{L^{p, \sigma} z_{*}}(x, 1)
$$

for $x \in B\left(0, \delta_{k}\right)$. It follows from (4.10), (4.11) and (4.12) that

$$
\left|\tilde{w}_{j}(x, t)\right| \leq C M_{k, i} \eta_{2}(t)\|\phi\|_{L^{p, \sigma}} \leq C M_{k, i}\|\phi\|_{L^{p, \sigma} z_{*}}(x, t)
$$

for $x \in \partial B\left(0, \delta_{k} \sqrt{t}\right)$ and $t \geq 1$. By (4.13) and (4.14), applying the comparison principle, we see that

$$
\left|\tilde{w}_{j}(x, t)\right| \leq C M_{k, i}\|\phi\|_{L^{p, \sigma} z_{*}}(x, t) \quad \text { for } x \in B\left(0, \delta_{k} \sqrt{t}\right) \text { and } t \geq 1 .
$$

This together with (4.11) and (4.12) implies that

$$
\left|\tilde{w}_{j}(x, t)\right| \leq C M_{k, i}\|\phi\|_{L^{p, \sigma}} \eta_{2}(t) \leq C M_{k, i} t^{-\frac{N}{2}-j} \frac{\left\|h_{0}\right\|_{L^{p^{\prime}, \sigma^{\prime}}(B(0, \sqrt{t}))}}{h_{k}(\delta \sqrt{t}) h_{0}(\sqrt{t})}\|\phi\|_{L^{p, \sigma}}
$$


for $x \in B\left(0, \delta_{k} \sqrt{t}\right)$ and $t \geq 1$. This implies that inequality (4.1) holds for $x \in B\left(0, \delta_{k} \sqrt{t}\right)$ and $t \geq 1$ in the cases when $\alpha>0$ and when $\alpha=0$ and $\beta<0$.

Assume either $\alpha<0$ or $\alpha=0, \beta \geq 0$. Let $L^{\prime} \geq 1$ be such that

$$
\tilde{\eta}(t):=t^{-\alpha}\left[\log \left(L^{\prime}+t\right)\right]^{\beta}
$$

is monotone increasing in $(1, \infty)$. Then $\tilde{\eta}$ is a supersolution to problem $\left(\mathrm{W}_{k}\right)$. Furthermore, similarly to (4.13) and (4.14), by (4.9), (4.10) and (4.11) we have

$$
\begin{aligned}
&\left|\tilde{w}_{j}(x, 1)\right| \leq C M_{k, i}\|\phi\|_{L^{p, \sigma}} \tilde{\eta}(1) \quad \text { for } \quad x \in B\left(0, \delta_{k}\right), \\
&\left|\tilde{w}_{j}(x, t)\right| \leq C M_{k, i}\|\phi\|_{L^{p, \sigma}} \tilde{\eta}(t) \quad \text { for } \quad(x, t) \in \partial B\left(0, \delta_{k} \sqrt{t}\right) \times(1, \infty) .
\end{aligned}
$$

Applying the comparison principle, we see that $\left|\tilde{w}_{j}(x, t)\right| \leq C M_{k, i}\|\phi\|_{L^{p, \sigma}} \tilde{\eta}(t)$ for $(x, t) \in$ $B\left(0, \delta_{k} \sqrt{t}\right) \times[1, \infty)$. This together with (4.11) implies that inequality (4.1) holds for $x \in$ $B\left(0, \delta_{k} \sqrt{t}\right)$ and $t \geq 1$ in the cases when $\alpha<0$ and when $\alpha=0, \beta<0$. Thus inequality (4.1) holds for $x \in B\left(0, \delta_{k} \sqrt{t}\right)$ and $t \geq 1$ for fixed $k \in\{0,1,2, \ldots\}$.

Step 3: We complete the proof of Proposition 4.1. Thanks to Steps 1 and 2, it suffices to prove inequality (4.1) for $x \in B(0, \delta \sqrt{t}), t \geq 1$ and large enough $k$. In this step the letter $C$ denotes generic positive constants independent of $k$.

Let $k_{*} \in\{0,1,2, \ldots\}$ be as in Proposition 2.2 (b) and $k \in\left\{k_{*}, k_{*}+1, \ldots\right\}$. Then $h_{k}$ is monotone increasing in $(1, \infty)$. We construct a supersolution to problem $\left(\mathrm{W}_{k}\right)$. Since

$$
\frac{d}{d t}\left[h_{k}(\sqrt{t})^{-1}\right]=-\frac{1}{2} t^{-\frac{1}{2}} h_{k}(\sqrt{t})^{-2}\left(\frac{d}{d r} h_{k}\right)(\sqrt{t})
$$

by Proposition 2.2 (b) we find $M_{3}>0$ such that

$$
-k M_{3}^{-1} t^{-1} h_{k}(\sqrt{t})^{-1} \geq \frac{d}{d t} h_{k}(\sqrt{t})^{-1} \geq-k M_{3} t^{-1} h_{k}(\sqrt{t})^{-1}
$$

for $t>0$ and $k \geq k_{*}$. Let $\tilde{\alpha}, \tilde{\beta} \in \mathbf{R}$ be such that

$$
t^{-\frac{N}{2}-j} \frac{\left\|h_{0}\right\|_{L^{p^{\prime}, \sigma^{\prime}}(B(0, \sqrt{t}))}}{h_{0}(\sqrt{t})} \asymp t^{-\tilde{\alpha}}[\log (1+t)]^{\tilde{\beta}} \quad \text { for } \quad t \geq 1 .
$$

Set

$$
M_{4}:=|\tilde{\alpha}|+|\tilde{\beta}|(\log 2)^{-1}+M_{3} .
$$

By Lemma 4.2 we take small enough $\tilde{\delta} \in(0, \delta]$ to obtain

$$
0 \leq(k+1) M_{4} t^{-1} f_{k}(|x|) \leq \frac{1}{2}
$$

for $x \in B(0, \tilde{\delta} \sqrt{t})$ and $t>0$, where $f_{k}$ is as in Lemma 4.2, Set

$$
\zeta_{k}(t):=t^{-\tilde{\alpha}}[\log (2+t)]^{\tilde{\beta}} h_{k}(\tilde{\delta} \sqrt{t})^{-1} \quad \text { for } \quad t>0 .
$$


Then, by (4.15) we have

$$
\begin{aligned}
& \frac{d}{d t} \zeta_{k}(t)=-\tilde{\alpha} t^{-1} \zeta_{k}(t)+\tilde{\beta}[\log (2+t)]^{-1}(2+t)^{-1} \zeta_{k}(t) \\
& \quad+\left.\tilde{\delta}^{2} t^{-\tilde{\alpha}}[\log (2+t)]^{\tilde{\beta}} \frac{d}{d \tau}\left[h_{k}(\sqrt{\tau})^{-1}\right]\right|_{\tau=\tilde{\delta}^{2} t} \\
& \geq-\left[|\tilde{\alpha}|+|\tilde{\beta}|(\log 2)^{-1}\right] t^{-1} \zeta_{k}(t)-k M_{3} t^{-\tilde{\alpha}-1}[\log (2+t)]^{\tilde{\beta}} h_{k}(\tilde{\delta} \sqrt{t})^{-1} \geq-k M_{4} t^{-1} \zeta_{k}(t)
\end{aligned}
$$

for $t>0$ and $k \geq k_{*}$. Similarly, taking sufficiently large $k_{*}$ if necessary, we see that

$$
\frac{d}{d t} \zeta_{k}(t) \leq 0 \text { for } t>0
$$

Set

$$
z_{*}(x, t):=2 \zeta_{k}(t)\left[1-(k+1) M_{4} t^{-1} f_{k}(|x|)\right] .
$$

By (4.17) and (4.18), taking small enough $\tilde{\delta}$ if necessary, we apply Lemma 4.2 to see that $z_{*}$ is a supersolution to problem $\left(\mathrm{W}_{k}\right)$ and

$$
2 \zeta_{k}(t) \geq z_{*}(x, t) \geq \zeta_{k}(t) \quad \text { for } \quad x \in B(0, \tilde{\delta} \sqrt{t}) \quad \text { and } \quad t>0 .
$$

Here $\tilde{\delta} \in(0, \delta]$ depends only on $M_{1}$ and $M_{4}$ and it is independent of $k$. On the other hand, by (4.8) with $\delta=\tilde{\delta}$ we have

$$
\left|\tilde{w}_{j}(x, 1)\right| \leq C M_{k, i}\|\phi\|_{L^{p, \sigma}} \frac{\zeta_{k}(1)}{h_{k}(\tilde{\delta}) \zeta_{k}(1)} \leq C M_{k, i}\|\phi\|_{L^{p, \sigma} \zeta_{k}(1) \leq C M_{k, i}\|\phi\|_{L^{p, \sigma}} z_{*}(x, 1)}
$$

for $x \in B(0, \tilde{\delta})$. On the other hand, by Lemma 4.1 and (4.16) we obtain

$$
\left|\tilde{w}_{j}(x, t)\right| \leq C M_{k, i} t^{-\frac{N}{2}-j} \frac{\left\|h_{0}\right\|_{L^{p^{\prime}, \sigma^{\prime}}(B(0, \sqrt{t})}}{h_{k}(\tilde{\delta} \sqrt{t}) h_{0}(\sqrt{t})}\|\phi\|_{L^{p, \sigma}} \leq C M_{k, i}\|\phi\|_{L^{p, \sigma}} \zeta(t)
$$

for $x \in \partial B(0, \tilde{\delta} \sqrt{t})$ and $t>0$. By (4.19), (4.20) and (4.21) we apply the comparison principle to obtain

$$
\left|\tilde{w}_{j}(x, t)\right| \leq C M_{k, i}\|\phi\|_{L^{p, \sigma} z_{*}}(x, t) \leq C M_{k, i}\|\phi\|_{L^{p, \sigma} \zeta(t)}
$$

for $x \in B(0, \tilde{\delta} \sqrt{t})$ and $t>0$. This together with (4.16) implies that inequality (4.1) holds for $x \in B(0, \tilde{\delta} \sqrt{t}), t \geq 1$ and $k \geq k_{*}$. Thus Proposition 4.1 follows.

\subsection{Estimates of spacial derivatives}

In this subsection, thanks to Proposition 4.1, we obtain the following proposition.

Proposition 4.2 Assume the same conditions as in Proposition 4.1. Set

$$
w_{k, i}(|x|, t):=\frac{v_{k, i}(|x|, t)}{h_{k}(|x|)} \quad \text { for } \quad(x, t) \in \mathbf{R}^{N} \times(0, \infty),
$$


where $k \in\{0,1,2, \ldots\}$ and $i \in\left\{1, \ldots, d_{k}\right\}$. Then, for any $j \in\{0,1,2, \ldots\}$,

$$
\partial_{t}^{j} w_{k, i}(|x|, t)=\partial_{t}^{j} w_{k, i}(0, t)+F_{k, i}^{j}(x, t), \quad(x, t) \in \mathbf{R}^{N} \times(0, \infty),
$$

where

$$
F_{k, i}^{j}(x, t):=\int_{0}^{|x|} s^{1-N} \nu_{k}(s)^{-1}\left(\int_{0}^{s} \tau^{N-1} \nu_{k}(\tau)\left[\partial_{t}^{j+1} w_{k, i}\right](\tau, t) d \tau\right) d s .
$$

Furthermore, there exists $C>0$ and $\delta \in(0,1]$ such that

$$
\begin{aligned}
& \left|\partial_{t}^{j} w_{k, i}(x, t)\right| \leq C M_{k, i} t^{-\frac{N}{2}-j} \frac{\left\|h_{0}\right\|_{L^{p^{\prime}, \sigma^{\prime}}(B(0, \sqrt{t}))}}{h_{k}(\delta \sqrt{t}) h_{0}(\sqrt{t})}\|\phi\|_{L^{p, \sigma}}, \\
& \left|\nabla^{\ell} F_{k, i}^{j}(x, t)\right| \leq C M_{k, i} t^{-\frac{N}{2}-j-1}|x|^{2-\ell} \frac{\left\|h_{0}\right\|_{L^{p^{\prime}, \sigma^{\prime}}(B(0, \sqrt{t}))}}{h_{k}(\delta \sqrt{t}) h_{0}(\sqrt{t})}\|\phi\|_{L^{p, \sigma}},
\end{aligned}
$$

for $x \in B(0, \delta \sqrt{t})$ and $t>0$, where $\ell \in\{0,1\}$.

Proof. Let $j \in\{0,1,2, \ldots\}$. By Proposition 4.1 we find $C>0$ and $\delta \in(0,1]$ such that

$$
\left|\partial_{t}^{j+1} w_{k, i}(x, t)\right| \leq C M_{k, i} t^{-\frac{N}{2}-j-1} \frac{\left\|h_{0}\right\|_{L^{p^{\prime}, \sigma^{\prime}}(B(0, \sqrt{t}))}}{h_{k}(\delta \sqrt{t}) h_{0}(\sqrt{t})}\|\phi\|_{L^{p, \sigma}}
$$

for $x \in B(0, \delta \sqrt{t}), t>0$ and $k \in\{0,1,2, \ldots\}$. This implies (4.23). Furthermore, combining (4.25) and Proposition 2.4 (b), we have

$$
\begin{aligned}
& \left.|| x\right|^{1-N} \nu_{k}(|x|)^{-1} \int_{0}^{|x|} \tau^{N-1} \nu_{k}(\tau)\left(\partial_{t}^{j+1} w_{k, i}\right)(\tau, t) d \tau \mid \\
& \quad \leq\left\|\partial_{t}^{j+1} w_{k, i}(t)\right\|_{L^{\infty}(B(0, \delta \sqrt{t}))} \cdot|x|^{1-N} \nu_{k}(|x|)^{-1} \int_{0}^{|x|} \tau^{N-1} \nu_{k}(\tau) d \tau \\
& \leq C(k+1)^{-1}|x|\left\|\partial_{t}^{j+1} w_{k, i}(t)\right\|_{L^{\infty}(B(0, \delta \sqrt{t}))}, \\
& \left|\nabla^{\ell} F_{k, i}^{j}(x, t)\right| \leq C(k+1)^{-1} M_{k, i} t^{-\frac{N}{2}-j-1}|x|^{2-\ell} \frac{\left\|h_{0}\right\|_{L^{p^{\prime}, \sigma^{\prime}}(B(0, \sqrt{t}))}}{h_{k}(\delta \sqrt{t}) h_{0}(\sqrt{t})}\|\phi\|_{L^{p, \sigma}},
\end{aligned}
$$

for $x \in B(0, \delta \sqrt{t}), t>0$ and $k \in\{0,1,2, \ldots\}$, where $\ell \in\{0,1\}$. Then $F_{k, i}^{j}$ is well-defined and (4.24) holds. Furthermore, it satisfies

$$
\frac{1}{\nu_{k}} \operatorname{div}\left(\nu_{k} \nabla F_{k, i}^{j}\right)=\partial_{t}^{j+1} w_{k, i} \quad \text { in } \quad \mathbf{R}^{N} \times(0, \infty) .
$$

Set

$$
\tilde{w}_{k, i}^{j}(|x|, t):=\partial_{t}^{j} w_{k, i}(|x|, t)-F_{k, i}^{j}(|x|, t) .
$$

Then it follows that

$$
\frac{1}{\nu_{k}} \operatorname{div}\left(\nu_{k} \nabla \tilde{w}_{k}\right)=0 \quad \text { in } \quad \mathbf{R}^{N} \times(0, \infty) .
$$


For any fixed $t>0$, set $z_{k, i}^{j}(r):=h_{k}(r) \tilde{w}_{k, i}^{j}(r, t)$ for $r>0$. Then, by (4.27) we see that $z_{k, i}^{j}$ satisfies

$$
\begin{aligned}
& \frac{d^{2}}{d r^{2}} z_{k, i}^{j}+\frac{N-1}{r} \frac{d}{d r} z_{k, i}^{j}-V_{k}(r) z_{k, i}^{j}=0 \quad \text { for } \quad r \in(0, \infty), \\
& \lim _{r \rightarrow+0} \frac{z_{k, i}^{j}(r)}{h_{k}(r)}=\tilde{w}_{k, i}^{j}(0, t)=\partial_{t}^{j} w_{k, i}(0, t) .
\end{aligned}
$$

By (1.4) we see that $z_{k, i}^{j}(r)=\partial_{t}^{j} w_{k, i}(0, t) h_{k}(r)$ for $r \in(0, \infty)$, that is,

$$
\partial_{t}^{j} w_{k, i}(|x|, t)-F_{k, i}^{j}(|x|, t)=\tilde{w}_{k, i}^{j}(|x|, t)=\partial_{t}^{j} w_{k, i}(0, t), \quad x \in \mathbf{R}^{N}
$$

for $t>0$. Thus relation (4.22) holds. Thus Proposition 4.2 follows.

\section{$5 \quad$ Proof of Theorem 1.1}

We complete the proof of Theorem 1.1, and prove Corollary 1.1.

Proof of Theorem 1.1. Theorem 1.1 with $\ell=0$ follows from Proposition 3.1 (b). It suffices to prove (1.6) with $\ell=1$.

Let $(p, q, \sigma, \theta) \in \Lambda$ and $\ell=1$. We can assume, without loss of generality, that $h_{0} \in$ $L^{p^{\prime}, \sigma^{\prime}}(B(0,1))$ and $\nabla h_{0} \in L^{q, \theta}(B(0,1))$. Then, by the definition of the Lorentz norm and Proposition 2.1 we see that $h_{0} \in L^{q, \theta}(B(0,1))$. These imply that

$$
h_{0} \in L^{p^{\prime}, \sigma^{\prime}}(B(0,1)) \cap L^{p, \sigma}(B(0,1)) \cap L^{q, \theta}(B(0,1)) .
$$

Let $\phi \in C_{c}\left(\mathbf{R}^{N}\right)$. We use the same notations as in (1.8), (1.9) and (1.10). By (1.1) we apply the regularity theorems for elliptic equations to obtain

$$
\left\|Q_{k, i}\right\|_{L^{\infty}\left(\mathbf{S}^{N-1}\right)}+\left\|\nabla Q_{k, i}\right\|_{L^{\infty}\left(\mathbf{S}^{N-1}\right)} \leq C\left(\omega_{k}+1\right)\left\|Q_{k, i}\right\|_{L^{2}\left(\mathbf{S}^{N-1}\right)} \leq C(k+1)^{2}
$$

for $\ell \in\{0,1\}, k \in\{0,1,2, \ldots\}$ and $i \in\left\{1, \ldots, d_{k}\right\}$. Since

$$
u_{k, i}(x, t)=w_{k, i}(x, t) h_{k}(|x|) Q_{k, i}\left(\frac{x}{|x|}\right)
$$

applying Proposition 4.2 with (5.1), we obtain

$$
\begin{aligned}
\left|\partial_{t}^{j} \nabla u_{k, i}(x, t)\right| \leq C & (k+1)^{4} t^{-\frac{N}{2}-j} \frac{\left\|h_{0}\right\|_{L^{p^{\prime}, \sigma^{\prime}}(B(0, \sqrt{t})}}{h_{k}(\delta \sqrt{t}) h_{0}(\sqrt{t})}\|\phi\|_{L^{p, \sigma}} \\
& \times\left\{t^{-1}|x| h_{k}(x)+\left|\nabla h_{k}(x)\right|+|x|^{-1} h_{k}(x)\right\}
\end{aligned}
$$

for $x \in B(0, \delta \sqrt{t}) \backslash\{0\}$ and $t>0$. Here $\delta$ is as in Proposition 4.2. By Propositions 2.1] and 2.2 we see that

$$
\left|\partial_{t}^{j} \nabla u_{k, i}(x, t)\right| \leq C(k+1)^{5} t^{-\frac{N}{2}-j} \frac{\left\|h_{0}\right\|_{L^{p^{\prime}, \sigma^{\prime}}(B(0, \sqrt{t}))}}{h_{k}(\delta \sqrt{t}) h_{0}(\sqrt{t})}\|\phi\|_{L^{p, \sigma}}|x|^{-1} h_{k}(x)
$$


for $x \in B(0, \delta \sqrt{t}) \backslash\{0\}$ and $t>0$. Set

$$
\tilde{u}(x, t):=u(x, t)-u_{0,1}(x, t)=\sum_{k=1}^{\infty} \sum_{i=1}^{d_{k}} u_{k, i}(x, t) .
$$

By (1.2), (1.10) and (5.3) we see that

$$
\begin{aligned}
& \left|\partial_{t}^{j} \nabla \tilde{u}(x, t)\right| \leq \sum_{k=1}^{\infty} \sum_{i=1}^{d_{k}}\left|\partial_{t}^{j} \nabla u_{k, i}(x, t)\right| \\
& \quad \leq C\|\phi\|_{L^{p, \sigma}} t^{-\frac{N}{2}-j}|x|^{-1} \frac{\left\|h_{0}\right\|_{L^{p^{\prime}, \sigma^{\prime}}(B(0, \sqrt{t}))}}{h_{0}(\sqrt{t})} \sum_{k=1}^{\infty} \sum_{i=1}^{d_{k}}(k+1)^{5} \frac{h_{k}(|x|)}{h_{k}(\delta \sqrt{t})} \\
& \quad \leq C\|\phi\|_{L^{p, \sigma}} t^{-\frac{N}{2}-j}|x|^{-1} \frac{\left\|h_{0}\right\|_{L^{p^{\prime}, \sigma^{\prime}}(B(0, \sqrt{t}))}}{h_{0}(\sqrt{t})} \sum_{k=1}^{\infty}(k+1)^{N+3} \frac{h_{k}(|x|)}{h_{k}(\delta \sqrt{t})}
\end{aligned}
$$

for $x \in B(0, \delta \sqrt{t}) \backslash\{0\}$ and $t>0$.

For $k \in\{1,2, \ldots\}$, set

$$
\iota_{k}(r):= \begin{cases}r^{A_{1, k}-A_{1,1}} & \text { for } \quad 0<r<1 \\ r^{A_{2, k}-A_{2,1}} & \text { for } \quad r \geq 1\end{cases}
$$

Then $\iota_{k}$ is monotone increasing in $(0, \infty)$. Furthermore, by Proposition 2.3 we have

$$
\frac{h_{k}(r)}{h_{1}(r)} \asymp \iota_{k}(r) \text { for } r>0 \text { and } k \in\{1,2, \ldots\} \text {. }
$$

Since $A_{i, k}-A_{i, 1}=k(1+o(1))$ as $k \rightarrow \infty$, where $i=1$, 2 , we find $\gamma>0$ such that

$$
A_{i, k}-A_{i, 1} \geq \frac{k}{2}-\gamma \quad \text { for } \quad k \in\{1,2, \ldots\}
$$

Then we see that $\iota_{k}(\epsilon r) \leq \epsilon^{\frac{k}{2}-\gamma} \iota_{k}(r)$ for $r>0$ and $\epsilon \in(0,1)$. This implies that

$$
\begin{aligned}
\frac{h_{k}(|x|)}{h_{k}(\delta \sqrt{t})} & =\frac{h_{1}(|x|)}{h_{1}(\delta \sqrt{t})} \frac{h_{k}(|x|)}{h_{1}(|x|)} \frac{h_{1}(\delta \sqrt{t})}{h_{k}(\delta \sqrt{t})} \\
& \leq C \frac{h_{1}(|x|)}{h_{1}(\delta \sqrt{t})} \frac{\iota_{k}(|x|)}{\iota_{k}(\delta \sqrt{t})} \leq C \frac{h_{1}(|x|)}{h_{1}(\delta \sqrt{t})} \frac{\iota_{k}(\epsilon \delta \sqrt{t})}{\iota_{k}(\delta \sqrt{t})} \leq C \epsilon^{\frac{k}{2}-\gamma} \frac{h_{1}(|x|)}{h_{1}(\delta \sqrt{t})}
\end{aligned}
$$

for $x \in B(0, \epsilon \delta \sqrt{t}) \backslash\{0\}, t>0, \epsilon \in(0,1)$ and $k \in\{1,2, \ldots\}$. Taking small enough $\epsilon>0$ if necessary, we see that

$$
\sum_{k=1}^{\infty}(k+1)^{N+3} \frac{h_{k}(|x|)}{h_{k}(\delta \sqrt{t})} \leq C \frac{h_{1}(|x|)}{h_{1}(\delta \sqrt{t})} \sum_{k=1}^{\infty} \epsilon^{\frac{k}{2}-\gamma}(k+1)^{N+3} \leq C_{\epsilon} \frac{h_{1}(|x|)}{h_{1}(\delta \sqrt{t})}
$$


for $x \in B(0, \epsilon \delta \sqrt{t})) \backslash\{0\}$ and $t>0$. Here $C_{\epsilon}$ is a positive constant depending on $\epsilon>0$. By (5.4) and (5.5) we obtain

$$
\left|\partial_{t}^{j} \nabla \tilde{u}(x, t)\right| \leq C C_{\epsilon}\|\phi\|_{L^{p, \sigma}} t^{-\frac{N}{2}-j}|x|^{-1} \frac{\left\|h_{0}\right\|_{L^{p^{\prime}, \sigma^{\prime}}(B(0, \sqrt{t}))}}{h_{0}(\sqrt{t})} \frac{h_{1}(|x|)}{h_{1}(\delta \sqrt{t})}
$$

for $x \in B(0, \epsilon \delta \sqrt{t}) \backslash\{0\}$ and $t>0$. On the other hand, by (5.2) and (5.3) we have

$$
\begin{aligned}
\left|\partial_{t}^{j} \nabla u_{0,1}(x, t)\right| & \leq C t^{-\frac{N}{2}-j-1}|x| \frac{\left\|h_{0}\right\|_{L^{p^{\prime}, \sigma^{\prime}}(B(0, \sqrt{t}))}}{h_{0}(\sqrt{t}) h_{0}(\delta \sqrt{t})}\|\phi\|_{L^{p, \sigma}}\left|h_{0}(x)\right| \\
& +C t^{-\frac{N}{2}-j} \frac{\left\|h_{0}\right\|_{L^{p^{\prime}, \sigma^{\prime}}(B(0, \sqrt{t})}}{h_{0}(\sqrt{t}) h_{0}(\delta \sqrt{t})}\|\phi\|_{L^{p, \sigma}}\left|\nabla h_{0}(x)\right| \\
& \leq C t^{-\frac{N}{2}-j} \frac{\left\|h_{0}\right\|_{L^{p^{\prime}, \sigma^{\prime}}(B(0, \sqrt{t}))}}{h_{0}(\sqrt{t}) h_{0}(\delta \sqrt{t})}\|\phi\|_{L^{p, \sigma}}\left[\left|\nabla h_{0}(x)\right|+t^{-\frac{1}{2}} h_{0}(|x|)\right]
\end{aligned}
$$

for $x \in B(0, \epsilon \delta \sqrt{t}) \backslash\{0\}$ and $t>0$. Therefore, combining Proposition 3.1 (a), (5.6) and (5.7), we obtain

$$
\begin{aligned}
& \left\|\partial_{t}^{j} \nabla u(t)\right\|_{L^{q, \theta}} \\
& \leq\left\|\partial_{t}^{j} \nabla u_{0,1}(t)\right\|_{L^{q, \theta}(B(0, \epsilon \delta \sqrt{t}))}+\left\|\partial_{t}^{j} \nabla \tilde{u}(t)\right\|_{L^{q, \theta}(B(0, \epsilon \delta \sqrt{t}))}+\left\|\partial_{t}^{j} \nabla u(t)\right\|_{L^{q, \theta}(B(0, \epsilon \delta \sqrt{t}))^{c}} \\
& \leq C t^{-\frac{N}{2}-j} \frac{\left\|h_{0}\right\|_{L^{p^{\prime}, \sigma^{\prime}}(B(0, \sqrt{t}))}}{h_{0}(\sqrt{t})}\|\phi\|_{L^{p, \sigma}} \\
& \quad \times\left[\frac{\left\|\nabla h_{0}\right\|_{L^{q, \theta}(B(0, \epsilon \delta \sqrt{t}))}}{h_{0}(\delta \sqrt{t})}+t^{-\frac{1}{2}} \frac{\left\|h_{0}\right\|_{L^{q, \theta}(B(0, \epsilon \delta \sqrt{t}))}}{h_{0}(\delta \sqrt{t})}+\frac{\left\|\tilde{h}_{1}\right\|_{L^{q, \theta}(B(0, \epsilon \delta \sqrt{t}))}}{h_{1}(\delta \sqrt{t})}\right]
\end{aligned}
$$

for $t>0$, where $\tilde{h}_{1}(x):=|x|^{-1} h_{1}(|x|)$. This together with (1.4) and (1.5) implies that

$$
\begin{aligned}
\left\|\partial_{t}^{j} \nabla e^{-t H}\right\|_{\left(L^{p, \sigma} \rightarrow L^{q, \theta}\right)} & \leq C t^{-\frac{N}{2}-j} \frac{\left\|h_{0}\right\|_{L^{p^{\prime}, \sigma^{\prime}}(B(0, \sqrt{t}))}}{h_{0}(\sqrt{t})} \\
& \times\left[\frac{\left\|\nabla h_{0}\right\|_{L^{q, \theta}(B(0, \sqrt{t}))}}{h_{0}(\sqrt{t})}+t^{-\frac{1}{2}} \frac{\left\|h_{0}\right\|_{L^{q, \theta}(B(0, \sqrt{t}))}}{h_{0}(\sqrt{t})}+\frac{\left\|\tilde{h}_{1}\right\|_{L^{q, \theta}(B(0, \sqrt{t}))}}{h_{1}(\sqrt{t})}\right]
\end{aligned}
$$

for $t>0$.

On the other hand, by Proposition 2.1 we find $R>0$ such that

$$
\begin{array}{lll}
h_{0}(|x|) \leq C|x|\left|\nabla h_{0}(|x|)\right| \leq C t^{\frac{1}{2}}\left|\nabla h_{0}(|x|)\right| & \text { if } & \lambda_{1} \neq 0, \\
h_{0}(|x|) \leq C & \text { if } & \lambda_{1}=0,
\end{array}
$$

for $x \in[B(0, R) \cap B(0, \sqrt{t})] \backslash\{0\}$. Furthermore, taking small enough $R>0$ if necessary, by (1.4) we see that

$$
\frac{h_{1}(r)}{h_{0}(r)} \leq C \frac{h_{1}(\sqrt{t})}{h_{0}(\sqrt{t})} \quad \text { for } \quad x \in B(0, \sqrt{t}) \cap B(0, R) .
$$


These imply that

$$
\begin{aligned}
& \frac{\tilde{h}_{1}(|x|)}{h_{1}(\sqrt{t})}=\frac{|x|^{-1} h_{1}(|x|)}{h_{1}(\sqrt{t})} \leq C|x|^{-1} \frac{h_{0}(|x|)}{h_{0}(\sqrt{t})} \leq C \frac{\left|\nabla h_{0}(|x|)\right|}{h_{0}(\sqrt{t})} \quad \text { if } \quad \lambda_{1} \neq 0, \\
& \frac{\tilde{h}_{1}(|x|)}{h_{1}(\sqrt{t})} \leq C|x|^{-1} \frac{|x|}{\sqrt{t}} \leq C t^{-\frac{1}{2}} \quad \text { if } \quad \lambda_{1}=0,
\end{aligned}
$$

for $x \in[B(0, \sqrt{t}) \cap B(0, R)] \backslash\{0\}$. By (5.8), (5.9) and (5.10) we obtain

$$
\left\|\partial_{t}^{j} \nabla e^{-t H}\right\|_{\left(L^{p, \sigma} \rightarrow L^{q, \theta}\right)} \leq C t^{-\frac{N}{2}-j} \frac{\left\|h_{0}\right\|_{L^{p^{\prime}, \sigma^{\prime}}(B(0, \sqrt{t}))}}{h_{0}(\sqrt{t})}\left[\frac{\left\|\nabla h_{0}\right\|_{L^{q, \theta}(B(0, \sqrt{t}))}}{h_{0}(\sqrt{t})}+t^{\frac{N}{2 q}-\frac{1}{2}}\right]
$$

for $t \in(0, \sqrt{R})$, which implies (1.6) for $t \in(0, \sqrt{R})$. Therefore Theorem 1.1 follows in the case when $0<t<\sqrt{R}$.

It remains to prove that (1.6) holds for $t \geq \sqrt{R}$. Similarly to (5.9), by Proposition 2.2 we find $R^{\prime} \in(R, \infty)$ such that

$$
\begin{aligned}
& h_{0}(|x|) \leq C \quad \text { if } \quad v_{0} \equiv 1 \text {, } \\
& h_{0}(|x|) \leq C|x|\left|\nabla h_{0}(|x|)\right| \leq C t^{\frac{1}{2}}\left|\nabla h_{0}(|x|)\right| \text { otherwise, }
\end{aligned}
$$

for $x \in B\left(0, R^{\prime}\right)^{c} \cap B(0, \sqrt{t})$. Furthermore, similarly to (5.10), taking large enough $R^{\prime}$ if necessary, by (1.5) we see that $h_{1}(r) / h_{0}(r) \asymp v_{1}(r) / v_{0}(r)$ and $v_{1}(r) / v_{0}(r)$ is monotone increasing in $\left(R^{\prime}, \infty\right)$ and obtain

$$
\begin{aligned}
& \frac{\tilde{h}_{1}(|x|)}{h_{1}(\sqrt{t})} \leq C|x|^{-1} \frac{|x|}{\sqrt{t}} \leq C t^{-\frac{1}{2}} \quad \text { if } \quad v_{0} \equiv 1, \\
& \frac{\tilde{h}_{1}(|x|)}{h_{1}(\sqrt{t})}=\frac{|x|^{-1} h_{1}(|x|)}{h_{1}(\sqrt{t})} \leq C|x|^{-1} \frac{h_{0}(|x|)}{h_{0}(\sqrt{t})} \leq C \frac{\left|\nabla h_{0}(|x|)\right|}{h_{0}(\sqrt{t})} \quad \text { otherwise, }
\end{aligned}
$$

for $x \in B\left(0, R^{\prime}\right)^{c} \cap B(0, \sqrt{t})$. Therefore, by (5.8), (5.11) and (5.12) we obtain

$$
\left\|\partial_{t}^{j} \nabla e^{-t H}\right\|_{\left(L^{p, \sigma} \rightarrow L^{q, \theta}\right)} \leq C t^{-\frac{N}{2}-j} \frac{\left\|h_{0}\right\|_{L^{p^{\prime}, \sigma^{\prime}}(B(0, \sqrt{t}))}}{h_{0}(\sqrt{t})}\left[\frac{\left\|\nabla h_{0}\right\|_{L^{q, \theta}(B(0, \sqrt{t}))}}{h_{0}(\sqrt{t})}+t^{\frac{N}{2 q}-\frac{1}{2}}\right]
$$

for $t \in[\sqrt{R}, \infty)$. This implies (1.6) for $t \in[\sqrt{R}, \infty)$. Thus Theorem 1.1 follows.

Proof of Corollary 1.1. Corollary 1.1 easily follows from Theorem 1.1 and Proposition 2.4(a).

\section{References}

[1] G. Barbatis, S. Filippas, and A. Tertikas, Critical heat kernel estimates for Schrödinger operators via HardySobolev inequalities, J. Funct. Anal. 208 (2004), 1-30.

[2] C. Bennett and R. Sharpley, Interpolation of operators, Pure and Applied Mathematics, vol. 129, Academic Press, Inc., Boston, MA, 1988. 
[3] I. Chavel and L. Karp, Large time behavior of the heat kernel: the parabolic $\lambda$-potential alternative, Comment. Math. Helv. 66 (1991), 541-556.

[4] D. Cruz-Uribe and C. Rios, Gaussian bounds for degenerate parabolic equations, J. Funct. Anal. 255 (2008), 283-312; Corrigendum in J. Funct. Anal. 267 (2014), 3507-3513.

[5] E. B. Davies, Heat kernels and spectral theory, Cambridge Tracts in Mathematics, vol. 92, Cambridge University Press, Cambridge, 1989.

[6] E. B. Davies and B. Simon, $L^{p}$ norms of noncritical Schrödinger semigroups, J. Funct. Anal. 102 (1991), 95-115.

[7] L. Grafakos, Classical Fourier analysis, 3rd ed., Graduate Texts in Mathematics, vol. 249, Springer, New York, 2014.

[8] A. Grigor'yan, Heat kernel and analysis on manifolds, AMS/IP Studies in Advanced Mathematics, vol. 47, American Mathematical Society, Providence, RI; International Press, Boston, MA, 2009.

[9] A. Grigor'yan and L. Saloff-Coste, Dirichlet heat kernel in the exterior of a compact set, Comm. Pure Appl. Math. 55 (2002), 93-133.

[10] A. Grigor'yan and L. Saloff-Coste, Stability results for Harnack inequalities, Ann. Inst. Fourier (Grenoble) 55 (2005), 825-890.

[11] N. Ioku, K. Ishige, and E. Yanagida, Sharp decay estimates of $L^{q}$-norms for nonnegative Schrödinger heat semigroups, J. Funct. Anal. 264 (2013), 2764-2783.

[12] _ Sharp decay estimates in Lorentz spaces for nonnegative Schrödinger heat semigroups, J. Math. Pures Appl. (9) 103 (2015), 900-923.

[13] K. Ishige, Gradient estimates for the heat equation in the exterior domains under the Neumann boundary condition, Differential Integral Equations 22 (2009), 401-410.

[14] K. Ishige and Y. Kabeya, Decay rates of the derivatives of the solutions of the heat equations in the exterior domain of a ball, J. Math. Soc. Japan 59 (2007), 861-898.

[15] $-L^{p}$ norms of nonnegative Schrödinger heat semigroup and the large time behavior of hot spots, J. Funct. Anal. 262 (2012), 2695-2733.

[16] K. Ishige, Y. Kabeya, and A. Mukai, Hot spots of solutions to the heat equation with inverse square potential, Appl. Anal. 98 (2019), 1843-1861.

[17] K. Ishige, Y. Kabeya, and E. M. Ouhabaz, The heat kernel of a Schrödinger operator with inverse square potential, Proc. Lond. Math. Soc. 115 (2017), 381-410.

[18] K. Ishige and A. Mukai, Large time behavior of solutions of the heat equation with inverse square potential, Discrete Contin. Dyn. Syst. 38 (2018), 4041-4069.

[19] K. Ishige and Y. Tateishi, Decay estimates for Schrödinger heat semigroup with inverse square potential. II, in preparation.

[20] O. A. Ladyženskaja, V. A. Solonnikov, and N. N. Ural'ceva, Linear and quasilinear equations of parabolic type, Translated from the Russian by S. Smith. Translations of Mathematical Monographs, Vol. 23, American Mathematical Society, Providence, R.I., 1968 (Russian).

[21] P. Li and S.-T. Yau, On the parabolic kernel of the Schrödinger operator, Acta Math. 156 (1986), $153-201$.

[22] G. M. Lieberman, Second order parabolic differential equations, World Scientific Publishing Co., Inc., River Edge, NJ, 1996.

[23] V. Liskevich and Z. Sobol, Estimates of integral kernels for semigroups associated with second-order elliptic operators with singular coefficients, Potential Anal. 18 (2003), 359-390.

[24] L. Angiuli and L. Lorenzi, On the estimates of the derivatives of solutions to nonautonomous Kolmogorov equations and their consequences, Riv. Math. Univ. Parma (N.S.) 7 (2016), 421-471.

[25] P. D. Milman and Yu. A. Semenov, Global heat kernel bounds via desingularizing weights, J. Funct. Anal. 212 (2004), 373-398; Corrigendum in J. Funct. Anal. 220 (2005), 238-239. 
[26] L. Moschini and A. Tesei, Harnack inequality and heat kernel estimates for the Schrödinger operator with Hardy potential, Atti Accad. Naz. Lincei Cl. Sci. Fis. Mat. Natur. Rend. Lincei (9) Mat. Appl. 16 (2005), 171-180 (2006).

[27] Parabolic Harnack inequality for the heat equation with inverse-square potential, Forum Math. 19 (2007), 407-427.

[28] M. Murata, Positive solutions and large time behaviors of Schrödinger semigroups, Simon's problem, J. Funct. Anal. 56 (1984), 300-310.

[29] S Structure of positive solutions to $(-\Delta+V) u=0$ in $\mathbf{R}^{n}$, Duke Math. J. 53 (1986), 869-943.

[30] E. M. Ouhabaz, Analysis of heat equations on domains, London Mathematical Society Monographs Series, vol. 31, Princeton University Press, Princeton, NJ, 2005.

[31] Y. Pinchover, On criticality and ground states of second order elliptic equations. II, J. Differential Equations 87 (1990), 353-364.

[32] L Large time behavior of the heat kernel and the behavior of the Green function near criticality for nonsymmetric elliptic operators, J. Funct. Anal. 104 (1992), 54-70.

[33] _ On positivity, criticality, and the spectral radius of the shuttle operator for elliptic operators, Duke Math. J. 85 (1996), 431-445.

[34] _ Large time behavior of the heat kernel, J. Funct. Anal. 206 (2004), 191-209.

[35] _ Some aspects of large time behavior of the heat kernel: an overview with perspectives, Mathematical physics, spectral theory and stochastic analysis, Oper. Theory Adv. Appl., vol. 232, Birkhäuser/Springer Basel AG, Basel, 2013, pp. 299-339.

[36] B. Simon, Large time behavior of the $L^{p}$ norm of Schrödinger semigroups, J. Functional Analysis 40 (1981), 66-83.

[37] Q. S. Zhang, Large time behavior of Schrödinger heat kernels and applications, Comm. Math. Phys. 210 (2000), 371-398.

[38] Global bounds of Schrödinger heat kernels with negative potentials, J. Funct. Anal. 182 (2001), 344-370. 